\title{
Regularity results and exponential growth for pullback attractors of a non-autonomous reaction-diffusion model with dynamical boundary conditions
}

\author{
María Anguiano, Pedro Marín-Rubio, José Real* \\ Dpto. de Ecuaciones Diferenciales y Análisis Numérico, Universidad de Sevilla \\ Apdo. de Correos 1160, 41080-Sevilla (Spain) \\ e-mails: anguiano@us.es pmr@us.es
}

May 7, 2014

\begin{abstract}
In this paper, we prove some regularity results for pullback attractors of a nonautonomous reaction-diffusion model with dynamical boundary conditions considered in [4]. Under certain assumptions of the nonlinear terms we show a regularity result for the unique solution of the problem. We establish a general result about boundedness of invariant sets for the associated evolution process in the norm of the domain of the spatial linear operator appearing in the equation. As a consequence, we deduce that the pullback attractors of the model are bounded in this domain norm. After that, under additional assumptions, some exponential growth results for pullback attractors when time goes to $-\infty$ are proved.
\end{abstract}

Keywords: Reaction-diffusion equations; dynamical boundary conditions; pullback attractors; tempered exponential growth.

Mathematics Subject Classifications (2010): 35K57, 35K61, 35B41, 37B55.

\section{Introduction and setting of the problem}

PDE problems with dynamic boundary conditions have the main characteristic of involving the time derivative of the unknown on the boundary of the domain. Although not so often considered in the literature as other boundary conditions (Dirichlet, Neumann, or Robin), dynamic boundary conditions also appear in a wide variety of applied problems.

Its use dates back at least to 1901 in the context of heat transfer in a solid in contact with a moving fluid (see the pioneering work by Peddie [36] in 1901, by March and Weaver [34] in 1928, by Peek [37] in 1929, by Langer [28] in 1932, and by Bauer [7] in 1953).

From the second half of the 20th century until today, they have been studied in many disciplines. Continuing with the topic of heat transfer between a domain and its boundary, see the more recent references $[45,33]$ and other variants, like heat transfer in two phase medium

${ }^{*}$ Prof. José Real deceased on 2012. M. A. and P.M.-R. would like to dedicate this paper to his memory. 
(e.g., cf. [39, 43, 11]; see [22] for a detailed study of a Cahn-Hilliard problem, a natural higher order generalization of the reaction-diffusion equation); problems in fluid dynamics (cf. [21] among others); diffusion phenomena (see [14]), in particular, diffusion in porous medium (e.g., cf. [20], beside the already cited reference [37]); probability theory and mathematical modeling in Biology (cf. [19]); thermoelasticity (cf. [26]); thermal energy storage devices (cf. [1]); chemical engineering (cf. [29]); semiconductor devices (cf. [42]), etcetera (see [8] for more problems and classical references). A detailed physical interpretation of this boundary condition can be found in [25]. For a more abstract point of view the reader is referred to $[16,5,6]$ among others.

The study of evolution equations with dynamic boundary conditions from the mathematical point of view dates back to 1961, when J. L. Lions [31] treated such equations and gave weak solutions by means of the variational method. Since then, this issue has been investigated to a large extent. In particular, the reaction-diffusion equation with dynamical boundary conditions arises in hydrodynamics and the heat transfer theory (see the above references), and this problem has strong background in mathematical physics. While some of the initial studies were devoted to existence results (e.g., cf. [27, 16, 17, 5, 38]), after that, the borderline (critical exponents) where blow-up phenomena appears or well-posedness holds was analyzed (e.g., cf. [6]), and some others papers are focused directly on well-posedness and the longtime behaviour under suitable dissipativity assumptions, including equilibria, description of convergence, different type of attractors, attracting w.r.t. several metrics, etcetera (cf. $[18,46$, $23,11,47,48,4])$.

By last in this brief introduction, let us recall that regularity issues concerning problems with dynamical boundary conditions have also been intensively analyzed. This is due to several facts. For instance, regularity properties allow to manage in different ways proofs of existence with different techniques, not only variational, but also by fixed points, using density arguments combined with approximating problems. Regularity features are also useful to deal with different flows in several phase-spaces, to improve attraction properties, and to implement more sophisticated numeric schemes. For example, regularity properties of several problems with dynamical boundary conditions, even of higher order, as wave equations and Cahn-Hilliard equations, with dynamic boundary conditions, have been addressed in [40, 41, 30].

Let us introduce the model we will be involved with in this paper. Let $\Omega \subset \mathbb{R}^{N}$ be a bounded domain with smooth enough boundary.

We consider the non-autonomous reaction-diffusion equation

$$
\frac{\partial u}{\partial t}-\Delta u+\kappa u+f(u)=h(t) \quad \text { in } \Omega \times(\tau, \infty),
$$

with the dynamical boundary condition

$$
\frac{\partial u}{\partial t}+\frac{\partial u}{\partial \vec{n}}+g(u)=\rho(t) \quad \text { on } \partial \Omega \times(\tau, \infty),
$$

where $\vec{n}$ is the outer normal to $\partial \Omega$, and the initial conditions

$$
\begin{gathered}
u(x, \tau)=u_{\tau}(x) \quad \text { for } x \in \Omega, \\
u(x, \tau)=\psi_{\tau}(x) \quad \text { for } x \in \partial \Omega,
\end{gathered}
$$

where $\tau \in \mathbb{R}$ is an initial time, and $\kappa>0, u_{\tau} \in L^{2}(\Omega), \psi_{\tau} \in L^{2}(\partial \Omega), h \in L_{l o c}^{2}\left(\mathbb{R} ; L^{2}(\Omega)\right)$, and $\rho \in L_{\text {loc }}^{2}\left(\mathbb{R} ; L^{2}(\partial \Omega)\right)$ are given. 
We also assume that the functions $f$ and $g \in C(\mathbb{R})$ are given, and satisfy that there exist constants $p \geq 2, q \geq 2, \alpha_{1}>0, \alpha_{2}>0, \beta>0$, and $l>0$, such that

$$
\begin{gathered}
\alpha_{1}|s|^{p}-\beta \leq f(s) s \leq \alpha_{2}|s|^{p}+\beta \quad \forall s \in \mathbb{R}, \\
\alpha_{1}|s|^{q}-\beta \leq g(s) s \leq \alpha_{2}|s|^{q}+\beta \quad \forall s \in \mathbb{R}, \\
(f(s)-f(r))(s-r) \geq-l(s-r)^{2} \text { and }(g(s)-g(r))(s-r) \geq-l(s-r)^{2} \quad \forall s, r \in \mathbb{R} .
\end{gathered}
$$

It is easy to deduce from above that there exists a constant $C>0$ such that

$$
|f(s)| \leq C\left(1+|s|^{p-1}\right), \quad|g(s)| \leq C\left(1+|s|^{q-1}\right)
$$

for all $s \in \mathbb{R}$.

Let us denote

$$
\mathcal{F}(s):=\int_{0}^{s} f(r) d r \quad \text { and } \quad \mathcal{G}(s):=\int_{0}^{s} g(r) d r .
$$

Then, there exist positive constants $\widetilde{\alpha}_{1}, \widetilde{\alpha}_{2}$, and $\widetilde{\beta}$ such that

$$
\widetilde{\alpha}_{1}|s|^{p}-\widetilde{\beta} \leq \mathcal{F}(s) \leq \widetilde{\alpha}_{2}|s|^{p}+\widetilde{\beta} \quad \forall s \in \mathbb{R},
$$

and

$$
\widetilde{\alpha}_{1}|s|^{q}-\widetilde{\beta} \leq \mathcal{G}(s) \leq \widetilde{\alpha}_{2}|s|^{q}+\widetilde{\beta} \quad \forall s \in \mathbb{R} .
$$

As we mentioned before, this model arises in different areas, especially in population growth, chemical reactions and heat conduction. For instance, in the case of a heat transfer in a medium $\Omega$, the equation (1) is a heat equation including an internal heat source. On the other hand, the heat flow from inside $\Omega$ to the boundary is $-\frac{\partial u}{\partial \vec{n}}$. The accumulation rate of heat on the boundary is $\frac{\partial u}{\partial t}$ so that, if we consider a source term on $\partial \Omega$, we have the dynamical boundary condition (2) (see the works by Constantin and Escher [13] and by Goldstein [25] for more details). Since the source terms may represent engineering or physic devices during the procedure (or noise perturbations, as in [12]), it is natural that they are time-depending, to indicate the time where they are acting with different intensities. This makes sense to consider some of the approaches of non-autonomous dynamical systems, as will be recalled below.

Before to continue with the setting of the problem, let us introduce some notation that will be useful in the sequel.

Let us consider the Hilbert space

$$
H:=L^{2}(\Omega) \times L^{2}(\partial \Omega),
$$

with the natural inner product $((v, \phi),(w, \varphi))_{H}=(v, w)_{\Omega}+(\phi, \varphi)_{\partial \Omega}$, which in particular induces the norm $|(\cdot, \cdot)|_{H}$ given by

$$
|(v, \phi)|_{H}^{2}=|v|_{\Omega}^{2}+|\phi|_{\partial \Omega}^{2}, \quad(v, \phi) \in H,
$$

where we denote by $(\cdot, \cdot)_{\Omega}$ (respectively, $(\cdot, \cdot)_{\partial \Omega}$ ) the inner product in $L^{2}(\Omega)$ (respectively, in $L^{2}(\partial \Omega)$ ), and by $|\cdot|_{\Omega}$ (respectively, $\left.|\cdot|_{\partial \Omega}\right)$ the associated norm. We will also denote $(\cdot, \cdot)_{\Omega}$ (respectively, $(\cdot, \cdot)_{\partial \Omega}$ ) the inner product in $\left(L^{2}(\Omega)\right)^{N}$, and the duality product between $L^{p^{\prime}}(\Omega)$ and $L^{p}(\Omega)$ (respectively, the duality product between $L^{q^{\prime}}(\partial \Omega)$ and $L^{q}(\partial \Omega)$ ). If $r \neq 2$, we will denote $|\cdot|_{r, \Omega}$ (respectively, $\left.|\cdot|_{r, \partial \Omega}\right)$ the norm in $L^{r}(\Omega)$ (respectively in $L^{r}(\partial \Omega)$ ). By 
$\|\cdot\|_{\Omega}$ we denote the norm in $H^{1}(\Omega)$, which is associated to the inner product $((\cdot, \cdot))_{\Omega}:=$ $(\nabla \cdot, \nabla \cdot)_{\Omega}+(\cdot, \cdot)_{\Omega}$.

We use the notation $\gamma_{0}$ for the trace operator $\left.u \mapsto u\right|_{\partial \Omega}$. The trace operator belongs to $\mathcal{L}\left(H^{1}(\Omega), H^{1 / 2}(\partial \Omega)\right)$, and we will use $\left\|\gamma_{0}\right\|$ to denote the norm of $\gamma_{0}$ in this space.

Finally, we will use $\|\cdot\|_{\partial \Omega}$ to denote the norm in $H^{1 / 2}(\partial \Omega)$, which is given by $\|\phi\|_{\partial \Omega}=$ $\inf \left\{\|v\|_{\Omega}: \gamma_{0}(v)=\phi\right\}$. We remember that with this norm, $H^{1 / 2}(\partial \Omega)$ is a Hilbert space.

A weak solution $(u, \psi)=\left(u\left(\cdot ; \tau, u_{\tau}, \psi_{\tau}\right), \psi\left(\cdot ; \tau, u_{\tau}, \psi_{\tau}\right)\right)$ to the problem (1)-(4) is a pair of functions $(u, \psi)$, satisfying $u \in C\left([\tau, \infty) ; L^{2}(\Omega)\right), \psi \in C\left([\tau, \infty) ; L^{2}(\partial \Omega)\right), u \in L^{2}\left(\tau, T ; H^{1}(\Omega)\right) \cap$ $L^{p}\left(\tau, T ; L^{p}(\Omega)\right)$ for all $T>\tau, \psi \in L^{2}\left(\tau, T ; H^{1 / 2}(\partial \Omega)\right) \cap L^{q}\left(\tau, T ; L^{q}(\partial \Omega)\right)$ for all $T>\tau$, and $\gamma_{0}(u(t))=\psi(t)$, a.e. $t \in(\tau, \infty)$, such that

$$
\begin{aligned}
& \frac{d}{d t}(u(t), v)_{\Omega}+\frac{d}{d t}\left(\psi(t), \gamma_{0}(v)\right)_{\partial \Omega}+(\nabla u(t), \nabla v)_{\Omega}+\kappa(u(t), v)_{\Omega}+(f(u(t)), v)_{\Omega} \\
& +\left(g\left(\gamma_{0}(u(t))\right), \gamma_{0}(v)\right)_{\partial \Omega}=(h(t), v)_{\Omega}+\left(\rho(t), \gamma_{0}(v)\right)_{\partial \Omega} \quad \text { in } \mathcal{D}^{\prime}(\tau, \infty),
\end{aligned}
$$

for all $v \in H^{1}(\Omega) \cap L^{p}(\Omega)$ such that $\gamma_{0}(v) \in L^{q}(\partial \Omega)$, and fulfills the initial conditions $u(\tau)=u_{\tau}$ and $\psi(\tau)=\psi_{\tau}$.

In Anguiano et al. [4] we discussed several issues concerning problem (1)-(4). We first proved the existence and uniqueness of solution using the monotonicity method, in the same way as in Chueshov and Schmalfuß [12].

A continuous dependence result with respect to initial data was addressed later. Therefore, the bi-parametric family of maps $U(t, \tau): H \rightarrow H$, with $\tau \leq t$, given by

$$
U(t, \tau)\left(u_{\tau}, \psi_{\tau}\right)=(u(t), \psi(t)),
$$

where $(u, \psi)=\left(u\left(\cdot ; \tau, u_{\tau}, \psi_{\tau}\right), \psi\left(\cdot ; \tau, u_{\tau}, \psi_{\tau}\right)\right)$ is the unique weak solution to (1)-(4), defines a process on $H$, which actually is continuous.

Then, we studied the asymptotic behaviour of this process in the framework of pullback attractors (as the deterministic analogy to the random attractor considered in [12]). Recall that a pullback attractor for the process $U$ (e.g., cf. $[9,10,15]$ ) is a family $\mathcal{A}=\{\mathcal{A}(t): t \in$ $\mathbb{R}\}$ of compact subsets of $H$ such that it satisfies the non-autonomous "invariance property" $U(t, \tau) \mathcal{A}(\tau)=\mathcal{A}(t)$ for all $\tau \leq t$; and it attracts in pullback sense elements from a certain given universe, i.e.,

$$
\lim _{\tau \rightarrow-\infty} \sup _{\left(u_{\tau}, \psi_{\tau}\right) \in B(\tau)} \inf _{(v, w) \in \mathcal{A}(t)}\left|U(t, \tau)\left(u_{\tau}, \psi_{\tau}\right)-(v, w)\right|_{H}=0
$$

for all $t \in \mathbb{R}$, and for any element $\{B(\tau)\}_{\tau \in \mathbb{R}} \subset \mathcal{P}(H)$ of the universe.

Namely, in [4, Theorem 23] we established the existence of two pullback attractors $\mathcal{A}_{\mathcal{D}_{F}^{H}}$ and $\mathcal{A}_{\mathcal{D}_{\mu}^{H}}$ (respectively for the universes of fixed bounded sets, and for another, parameterized in time, given by a tempered condition), assuming that $h$ and $\rho$ satisfy

$$
\int_{-\infty}^{0} e^{\mu s}\left(|h(s)|_{\Omega}^{2}+|\rho(s)|_{\partial \Omega}^{2}\right) d s<\infty
$$

where $\mu \in\left(0,2 \lambda_{1}\right), \lambda_{1}$ was given by (41) in [4], the first eigenvalue of a linear operator $A_{1}$ (recalled below). Also, using [35] and under additional assumptions on $h$ and $\rho$ we proved that $\mathcal{A}_{\mathcal{D}_{F}^{H}}(t)=\mathcal{A}_{\mathcal{D}_{\mu}^{H}}(t)$ for all $t \in \mathbb{R}$. Moreover, these two pullback attractors satisfy the same tempered growth in norm $L^{2}(\Omega) \times L^{2}(\partial \Omega)$ of the second universe,

$$
\lim _{\tau \rightarrow-\infty} e^{\mu \tau} \sup _{(v, \phi) \in \mathcal{A}(\tau)}\left(|v|_{\Omega}^{2}+|\phi|_{\partial \Omega}^{2}\right)=0 .
$$


However, as far as we know, there are no results in the literature concerning regularity and exponential growth of the pullback attractors as we will consider in this paper (for similar results for the reaction-diffusion equations with zero Dirichlet boundary condition see $[2,3]$, and for the Navier-Stokes equations see [24]). As commented before, the regularity results on the solutions and the attractors (that we obtain here) might be useful in the future in order to implement new methods to seek solutions of more general problems by different arguments, to gain attraction in higher norms, or for numerical purposes.

The structure of the paper is as follows. In Section 2, under an additional assumption relating the nonlinear terms $f$ and $g$, we establish a regularity result for the unique solution to problem (1)-(4). This relation among $f$ and $g$ (cf. Theorem 4) forces to take $q=p$, which will be assumed here on. In Section 3 we prove some results which, in particular, imply that under suitable assumptions, any pullback attractor $\mathcal{A}$ satisfies that any section $\mathcal{A}(t)$ or union of sections for a finite time-interval are bounded subsets of $\left(H^{1}(\Omega) \times H^{1 / 2}(\partial \Omega)\right) \cap\left(L^{p}(\Omega) \times L^{p}(\partial \Omega)\right)$ and also in the norm of the domain of $A_{1}, D\left(A_{1}\right)$. This result implies the pullback asymptotic smoothing effect of the model in the sense that the solutions eventually become more regular than the initial data. Finally, in Section 4 we prove two exponential growth results for these pullback attractors, firstly in the norm $\left(H^{1}(\Omega) \times H^{1 / 2}(\partial \Omega)\right) \cap\left(L^{p}(\Omega) \times L^{p}(\partial \Omega)\right)$, and later, under appropriate additional assumptions, in the norm of $D\left(A_{1}\right)$.

\section{A regularity result}

In this section we prove a regularity result for the weak solution to (1)-(4), whose existence and uniqueness is guaranteed in [4].

Let us consider the space

$$
V_{1}:=\left\{\left(v, \gamma_{0}(v)\right): v \in H^{1}(\Omega)\right\}
$$

We note that $V_{1}$ is a closed vector subspace of $H^{1}(\Omega) \times H^{1 / 2}(\partial \Omega)$, and therefore, with the norm $\|(\cdot, \cdot)\|_{V_{1}}$ given by

$$
\left\|\left(v, \gamma_{0}(v)\right)\right\|_{V_{1}}^{2}=\|v\|_{\Omega}^{2}+\left\|\gamma_{0}(v)\right\|_{\partial \Omega}^{2}, \quad\left(v, \gamma_{0}(v)\right) \in V_{1},
$$

$V_{1}$ is a Hilbert space. On the other hand, $V_{1}$ is densely embedded in $H$.

Now, on $V_{1}$ we define a continuous symmetric linear operator $A_{1}: V_{1} \rightarrow V_{1}^{\prime}$, given by

$$
\left\langle A_{1}\left(\left(v, \gamma_{0}(v)\right)\right),\left(w, \gamma_{0}(w)\right)\right\rangle=(\nabla v, \nabla w)_{\Omega}+\kappa(v, w)_{\Omega}, \quad \forall v, w \in H^{1}(\Omega) .
$$

Remark 1 The domain of the operator $A_{1}$ can be described as follows (which gives sense to the natural definition $\left.D\left(A_{1}\right)=\left\{\left(u, \gamma_{0}(u)\right) \in V_{1}: A_{1}\left(\left(u, \gamma_{0}(u)\right)\right) \in H\right\}\right)$. Let $(w, \psi) \in H$. Assume that $\left(u, \gamma_{0}(u)\right) \in V_{1}$ solves the equation

$$
A_{1}\left(\left(u, \gamma_{0}(u)\right)\right)=(w, \psi) \text {. }
$$

Then (see [5]) $\left(u, \gamma_{0}(u)\right)$, where $u \in H^{1}(\Omega)$, is a weak (variational) solution to the problem

$$
\left\{\begin{aligned}
-\Delta u+\kappa u & =w & & \text { in } \Omega \\
\frac{\partial u}{\partial \vec{n}} & =\psi & & \text { on } \partial \Omega .
\end{aligned}\right.
$$


Then, we may denote

$$
D\left(A_{1}\right)=\left\{\left(v, \gamma_{0}(v)\right) \in V_{1}: \Delta v \in L^{2}(\Omega), \frac{\partial v}{\partial \vec{n}} \in L^{2}(\partial \Omega)\right\},
$$

with the scalar product $(v, w)_{D\left(A_{1}\right)}=\left(A_{1} v, A_{1} w\right)_{H}$ for any $v$ and $w \in D\left(A_{1}\right)$, which induces the norm $\|(\cdot, \cdot)\|_{D\left(A_{1}\right)}$ given by $\left\|\left(v, \gamma_{0}(v)\right)\right\|_{D\left(A_{1}\right)}^{2}=\left|A_{1}\left(\left(v, \gamma_{0}(v)\right)\right)\right|_{H}^{2}$.

Let us observe that the space $H^{1}(\Omega) \times H^{1 / 2}(\partial \Omega)$ is compactly imbedded in $H$, and therefore, for the symmetric and coercive linear continuous operator $A_{1}: V_{1} \rightarrow V_{1}^{\prime}$, there exists a nondecreasing sequence $0<\lambda_{1} \leq \lambda_{2} \leq \ldots$ of eigenvalues associated to the operator $A_{1}$ with $\lim _{j \rightarrow \infty} \lambda_{j}=\infty$, and there exists a Hilbert basis of $H,\left\{\left(w_{j}, \gamma_{0}\left(w_{j}\right)\right): j \geq 1\right\} \subset D\left(A_{1}\right)$, with $\operatorname{span}\left\{\left(w_{j}, \gamma_{0}\left(w_{j}\right)\right): j \geq 1\right\}$ densely embedded in $V_{1}$, such that

$$
A_{1}\left(\left(w_{j}, \gamma_{0}\left(w_{j}\right)\right)\right)=\lambda_{j}\left(w_{j}, \gamma_{0}\left(w_{j}\right)\right) \quad \forall j \geq 1 .
$$

Remark 2 It can be proved that $\operatorname{span}\left\{\left(w_{j}, \gamma_{0}\left(w_{j}\right)\right): j \geq 1\right\}$ is densely embedded in $V_{1} \cap$ $\left(L^{p}(\Omega) \times L^{p}(\partial \Omega)\right)$.

Taking into account the above facts, we denote by

$$
\left(u_{m}(t), \gamma_{0}\left(u_{m}(t)\right)\right)=\left(u_{m}\left(t ; \tau, u_{\tau}, \psi_{\tau}\right), \gamma_{0}\left(u_{m}\left(t ; \tau, u_{\tau}, \psi_{\tau}\right)\right)\right)
$$

the Galerkin approximation of the solution $\left(u\left(t ; \tau, u_{\tau}, \psi_{\tau}\right), \gamma_{0}\left(u\left(t ; \tau, u_{\tau}, \psi_{\tau}\right)\right)\right)$ to (1)-(4) for each integer $m \geq 1$, which is given by

$$
\left(u_{m}(t), \gamma_{0}\left(u_{m}(t)\right)\right)=\sum_{j=1}^{m} \delta_{m j}(t)\left(w_{j}, \gamma_{0}\left(w_{j}\right)\right),
$$

and is the solution of

$$
\begin{aligned}
& \frac{d}{d t}\left(\left(u_{m}(t), \gamma_{0}\left(u_{m}(t)\right)\right),\left(w_{j}, \gamma_{0}\left(w_{j}\right)\right)\right)_{H}+\left\langle A_{1}\left(\left(u_{m}(t), \gamma_{0}\left(u_{m}(t)\right)\right)\right),\left(w_{j}, \gamma_{0}\left(w_{j}\right)\right)\right\rangle \\
& +\left(f\left(u_{m}(t)\right), w_{j}\right)_{\Omega}+\left(g\left(\gamma_{0}\left(u_{m}(t)\right)\right), \gamma_{0}\left(w_{j}\right)\right)_{\partial \Omega} \\
= & \left(h(t), w_{j}\right)_{\Omega}+\left(\rho(t), \gamma_{0}\left(w_{j}\right)\right)_{\partial \Omega}, \quad j=1, \ldots, m,
\end{aligned}
$$

with initial data

$$
\left(u_{m}(\tau), \gamma_{0}\left(u_{m}(\tau)\right)\right)=\left(u_{\tau_{m}}, \gamma_{0}\left(u_{\tau_{m}}\right)\right)
$$

where

$$
\delta_{m j}(t)=\left(u_{m}(t), w_{j}\right)_{\Omega}+\left(\gamma_{0}\left(u_{m}(t)\right), \gamma_{0}\left(w_{j}\right)\right)_{\partial \Omega}
$$

and $\left(u_{\tau_{m}}, \gamma_{0}\left(u_{\tau_{m}}\right)\right) \in \operatorname{span}\left\{\left(w_{j}, \gamma_{0}\left(w_{j}\right)\right): j=1, \ldots, m\right\}$ converge (when $\left.m \rightarrow \infty\right)$ to $\left(u_{\tau}, \psi_{\tau}\right)$ in a suitable sense which will be specified below.

Next result gives some preliminar (and standard) uniform estimates for the Galerkin approximations defined above when the initial data are suitably chosen. We include its proof for clarity since it will be used in the sequel.

Lemma 3 Under the given assumptions on $\kappa, f, g, h$, and $\rho$, consider a sequence $\left\{\left(u_{\tau_{m}}, \psi_{\tau_{m}}\right)\right\}$ bounded in $H$ with each $\left(u_{\tau_{m}}, \psi_{\tau_{m}}\right) \in \operatorname{span}\left\{\left(w_{j}, \gamma_{0}\left(w_{j}\right)\right): j=1, \ldots, m\right\}$. Then, the sequence of corresponding solutions to the Galerkin scheme (9)-(11) with these initial data is bounded in $L^{2}\left(\tau, T ; V_{1}\right) \cap L^{p}\left(\tau, T ; L^{p}(\Omega) \times L^{p}(\partial \Omega)\right)$ for all $T>\tau$. 
Proof. Multiplying by $\delta_{m j}$ in (10), and summing from $j=1$ to $m$, we obtain

$$
\begin{aligned}
& \frac{1}{2} \frac{d}{d r}\left|\left(u_{m}(r), \gamma_{0}\left(u_{m}(r)\right)\right)\right|_{H}^{2}+\left\langle A_{1}\left(\left(u_{m}(r), \gamma_{0}\left(u_{m}(r)\right)\right)\right),\left(u_{m}(r), \gamma_{0}\left(u_{m}(r)\right)\right)\right\rangle \\
& +\left(f\left(u_{m}(r)\right), u_{m}(r)\right)_{\Omega}+\left(g\left(\gamma_{0}\left(u_{m}(r)\right)\right), \gamma_{0}\left(u_{m}(r)\right)\right)_{\partial \Omega} \\
= & \left(h(r), u_{m}(r)\right)_{\Omega}+\left(\rho(r), \gamma_{0}\left(u_{m}(r)\right)\right)_{\partial \Omega}, \quad \text { a.e. } r>\tau .
\end{aligned}
$$

Using the properties of $f$ and $g$,

$$
\begin{aligned}
-\left(f\left(u_{m}(r)\right), u_{m}(r)\right)_{\Omega} & \leq \int_{\Omega}\left(\beta-\alpha_{1}\left|u_{m}(r)\right|^{p}\right) d x \\
& =\beta|\Omega|-\alpha_{1}\left|u_{m}(r)\right|_{p, \Omega}^{p} \quad \forall r \geq \tau,
\end{aligned}
$$

and

$$
\begin{aligned}
-\left(g\left(\gamma_{0}\left(u_{m}(r)\right)\right), \gamma_{0}\left(u_{m}(r)\right)\right)_{\partial \Omega} & \leq \int_{\partial \Omega}\left(\beta-\alpha_{1}\left|\gamma_{0}\left(u_{m}(r)\right)\right|^{p}\right) d \sigma(x) \\
& =\beta|\partial \Omega|-\alpha_{1}\left|\gamma_{0}\left(u_{m}(r)\right)\right|_{p, \partial \Omega}^{p} \quad \forall r \geq \tau .
\end{aligned}
$$

On the other hand,

$$
\left(h(r), u_{m}(r)\right)_{\Omega} \leq \frac{1}{\kappa}|h(r)|_{\Omega}^{2}+\frac{\kappa}{4}\left|u_{m}(r)\right|_{\Omega}^{2}, \quad \text { a.e. } r>\tau,
$$

and as $\left|\gamma_{0}\left(u_{m}(r)\right)\right|_{\partial \Omega} \leq\left\|\gamma_{0}\right\|\left\|u_{m}(r)\right\|_{\Omega}$, we have

$$
\begin{aligned}
\left(\rho(r), \gamma_{0}\left(u_{m}(r)\right)\right)_{\partial \Omega} & \leq \frac{\left\|\gamma_{0}\right\|^{2}}{2 \min \{1, \kappa / 2\}}|\rho(r)|_{\partial \Omega}^{2}+\frac{\min \{1, \kappa / 2\}}{2\left\|\gamma_{0}\right\|^{2}}\left|\gamma_{0}\left(u_{m}(r)\right)\right|_{\partial \Omega}^{2} \\
& \leq \frac{\left\|\gamma_{0}\right\|^{2}}{2 \min \{1, \kappa / 2\}}|\rho(r)|_{\partial \Omega}^{2}+\frac{1}{2} \min \{1, \kappa / 2\}\left\|u_{m}(r)\right\|_{\Omega}^{2}, \quad \text { a.e. } r>\tau .
\end{aligned}
$$

From above and the inequality (16) of [4], we deduce

$$
\begin{aligned}
& \frac{d}{d r}\left|\left(u_{m}(r), \gamma_{0}\left(u_{m}(r)\right)\right)\right|_{H}^{2}+\frac{\min \{1, \kappa\}}{1+\left\|\gamma_{0}\right\|^{2}}\left\|\left(u_{m}(r), \gamma_{0}\left(u_{m}(r)\right)\right)\right\|_{V_{1}}^{2} \\
& +2 \alpha_{1}\left|u_{m}(r)\right|_{p, \Omega}^{p}+2 \alpha_{1}\left|\gamma_{0}\left(u_{m}(r)\right)\right|_{p, \partial \Omega}^{p} \\
\leq & 2 \beta(|\Omega|+|\partial \Omega|)+\frac{2}{\kappa}|h(r)|_{\Omega}^{2}+\frac{\left\|\gamma_{0}\right\|^{2}}{\min \{1, \kappa / 2\}}|\rho(r)|_{\partial \Omega}^{2}, \quad \text { a.e. } r>\tau .
\end{aligned}
$$

Integrating between $\tau$ and $T$, the result follows.

Now we may establish a regularity result for the solution to the problem.

Theorem 4 Suppose that in addition to the assumptions on the data of the problem, we have that $p=q \geq 2$, that $f, g \in \mathcal{C}^{1}(\mathbb{R})$ and that there exists a constant $c_{f g}>0$ such that

$$
|f(s)-g(s)| \leq c_{f g}(1+|s|) \quad \forall s \in \mathbb{R},
$$

(which is only possible if $p=q$ ). Then, for any initial condition $\left(u_{\tau}, \psi_{\tau}\right) \in V_{1} \cap\left(L^{p}(\Omega) \times\right.$ $L^{p}(\partial \Omega)$ ), the weak solution to (1)-(4), whose existence and uniqueness is guaranteed in [4], satisfies in addition that $(u, \psi) \in C\left([\tau, T] ; V_{1}\right) \cap L^{2}\left(\tau, T ; D\left(A_{1}\right)\right) \quad \forall T>\tau$. 
Proof. We first note that under the conditions imposed we have that

$$
f^{\prime}(s) \geq-l, \quad g^{\prime}(s) \geq-l \quad \forall s \in \mathbb{R} .
$$

Let $\left(u_{\tau}, \psi_{\tau}\right) \in V_{1} \cap\left(L^{p}(\Omega) \times L^{p}(\partial \Omega)\right)$. For all $m \geq 1$, by Remark 2 , there exists $\left(u_{\tau_{m}}, \gamma_{0}\left(u_{\tau_{m}}\right)\right) \in$ $\operatorname{span}\left\{\left(w_{j}, \gamma_{0}\left(w_{j}\right)\right): 1 \leq j \leq m\right\}$, such that $\left\{\left(u_{\tau_{m}}, \gamma_{0}\left(u_{\tau_{m}}\right)\right)\right\}$ converges to $\left(u_{\tau}, \psi_{\tau}\right)$ in $V_{1}$ and in $L^{p}(\Omega) \times L^{p}(\partial \Omega)$ (and therefore in $L^{2}(\Omega) \times L^{2}(\partial \Omega)$ ).

For each integer $m \geq 1$, we consider the sequence $\left\{\left(u_{m}, \gamma_{0}\left(u_{m}\right)\right)\right\}$ defined by (9)-(11) with these initial data.

In order to prove the result, we will show firstly that the Galerkin approximations are uniformly bounded in $L^{\infty}\left(\tau, T ; V_{1} \cap L^{p}(\Omega) \times L^{p}(\partial \Omega)\right)$ with derivatives uniformly bounded in $L^{2}(\tau, T ; H)$ (and therefore the solution too) for all $T>\tau$.

Secondly, we will check that this sequence (and again the solution) is also bounded in $L^{2}\left(\tau, T ; D\left(A_{1}\right)\right)$ for all $T>\tau$, whence the result follows.

Step 1. The sequence $\left\{\left(u_{m}, \gamma_{0}\left(u_{m}\right)\right)\right\}$ is bounded in $L^{\infty}\left(\tau, T ; V_{1} \cap L^{p}(\Omega) \times L^{p}(\partial \Omega)\right)$ and their derivatives $\left\{\left(u_{m}^{\prime}, \gamma_{0}\left(u_{m}^{\prime}\right)\right)\right\}$ are bounded in $L^{2}(\tau, T ; H)$ for all $T>\tau$.

Multiplying by the derivative $\delta_{m j}^{\prime}$ in (10), and summing from $j=1$ to $m$, we obtain

$$
\begin{aligned}
& \left|\left(u_{m}^{\prime}(r), \gamma_{0}\left(u_{m}^{\prime}(r)\right)\right)\right|_{H}^{2}+\frac{1}{2} \frac{d}{d r}\left(\left\langle A_{1}\left(\left(u_{m}(r), \gamma_{0}\left(u_{m}(r)\right)\right)\right),\left(u_{m}(r), \gamma_{0}\left(u_{m}(r)\right)\right)\right\rangle\right) \\
& +\left(f\left(u_{m}(r)\right), u_{m}^{\prime}(r)\right)_{\Omega}+\left(g\left(\gamma_{0}\left(u_{m}(r)\right)\right), \gamma_{0}\left(u_{m}^{\prime}(r)\right)\right)_{\partial \Omega} \\
= & \left(h(r), u_{m}^{\prime}(r)\right)_{\Omega}+\left(\rho(r), \gamma_{0}\left(u_{m}^{\prime}(r)\right)\right)_{\partial \Omega}, \quad \text { a.e. } r>\tau .
\end{aligned}
$$

We observe that

$$
\left(f\left(u_{m}(r)\right), u_{m}^{\prime}(r)\right)_{\Omega}=\frac{d}{d r} \int_{\Omega} \mathcal{F}\left(u_{m}(r)\right) d x
$$

and

$$
\left(g\left(\gamma_{0}\left(u_{m}(r)\right)\right), \gamma_{0}\left(u_{m}^{\prime}(r)\right)\right)_{\partial \Omega}=\frac{d}{d r} \int_{\partial \Omega} \mathcal{G}\left(\gamma_{0}\left(u_{m}(r)\right)\right) d \sigma(x)
$$

for all $r \geq \tau$. Then, we deduce

$$
\begin{aligned}
& \left|\left(u_{m}^{\prime}(r), \gamma_{0}\left(u_{m}^{\prime}(r)\right)\right)\right|_{H}^{2}+\frac{1}{2} \frac{d}{d r}\left(\left\langle A_{1}\left(\left(u_{m}(r), \gamma_{0}\left(u_{m}(r)\right)\right)\right),\left(u_{m}(r), \gamma_{0}\left(u_{m}(r)\right)\right)\right\rangle\right) \\
\leq & \frac{1}{2}|h(r)|_{\Omega}^{2}+\frac{1}{2}\left|u_{m}^{\prime}(r)\right|_{\Omega}^{2}+\frac{1}{2}|\rho(r)|_{\partial \Omega}^{2}+\frac{1}{2}\left|\gamma_{0}\left(u_{m}^{\prime}(r)\right)\right|_{\partial \Omega}^{2} \\
& -\frac{d}{d r} \int_{\Omega} \mathcal{F}\left(u_{m}(r)\right) d x-\frac{d}{d r} \int_{\partial \Omega} \mathcal{G}\left(\gamma_{0}\left(u_{m}(r)\right)\right) d \sigma(x), \quad \text { a.e. } r>\tau .
\end{aligned}
$$

Integrating now between $\tau$ and $t$, and taking into account the definition of $A_{1}$ (cf. inequality (16) of [4]), (5) and (6), we obtain that

$$
\begin{aligned}
& \int_{\tau}^{t}\left|\left(u_{m}^{\prime}(\theta), \gamma_{0}\left(u_{m}^{\prime}(\theta)\right)\right)\right|_{H}^{2} d \theta+\frac{\min \{1, \kappa\}}{1+\left\|\gamma_{0}\right\|^{2}}\left\|\left(u_{m}(t), \gamma_{0}\left(u_{m}(t)\right)\right)\right\|_{V_{1}}^{2} \\
& +2 \widetilde{\alpha}_{1}\left|u_{m}(t)\right|_{p, \Omega}^{p}+2 \widetilde{\alpha}_{1}\left|\gamma_{0}\left(u_{m}(t)\right)\right|_{p, \partial \Omega}^{p} \\
\leq & \max \{1, \kappa\}\left\|\left(u_{m}(\tau), \gamma_{0}\left(u_{m}(\tau)\right)\right)\right\|_{V_{1}}^{2}+\int_{\tau}^{t}\left(|h(\theta)|_{\Omega}^{2}+|\rho(\theta)|_{\partial \Omega}^{2}\right) d \theta \\
& +2 \widetilde{\alpha}_{2}\left|u_{m}(\tau)\right|_{p, \Omega}^{p}+2 \widetilde{\alpha}_{2}\left|\gamma_{0}\left(u_{m}(\tau)\right)\right|_{p, \partial \Omega}^{p}+4 \tilde{\beta}(|\Omega|+|\partial \Omega|)
\end{aligned}
$$


for all $\tau \leq t$.

As long as the sequence $\left\{\left(u_{m}(\tau), \gamma_{0}\left(u_{m}(\tau)\right)\right)\right\}$ is bounded in $V_{1}$ and in $L^{p}(\Omega) \times L^{p}(\partial \Omega)$, Step 1 is proved.

Moreover, taking into account the uniqueness of solution to (1)-(4) and using Ascoli-Arzelà Theorem, it is not difficult to conclude that the sequence $\left\{\left(u_{m}, \gamma_{0}\left(u_{m}\right)\right)\right\}$ converges weakly-star in $L^{\infty}\left(\tau, T ; L^{p}(\Omega) \times L^{p}(\partial \Omega)\right)$ and in $L^{\infty}\left(\tau, T ; V_{1}\right)$ to the solution $\left(u, \gamma_{0}(u)\right)$ to (1)-(4), and we also obtain that $\left(u^{\prime}, \gamma_{0}\left(u^{\prime}\right)\right) \in L^{2}(\tau, T ; H)$.

Step 2. The sequence $\left\{\left(u_{m}, \gamma_{0}\left(u_{m}\right)\right)\right\}$ is bounded in $L^{2}\left(\tau, T ; D\left(A_{1}\right)\right)$.

In this way, by the above arguments we will have that $\left(u, \gamma_{0}(u)\right) \in L^{2}\left(\tau, T ; D\left(A_{1}\right)\right)$. After that, as $\left(u^{\prime}, \gamma_{0}\left(u^{\prime}\right)\right) \in L^{2}(\tau, T ; H)$, by the energy equality (see [44]), we can deduce that $\left(u, \gamma_{0}(u)\right) \in C\left([\tau, T] ; V_{1}\right)$ and the theorem will be proved.

Multiplying in (10) by $\lambda_{j} \delta_{m j}$, where $\lambda_{j}$ is the eigenvalue associated to the eigenfunction $\left(w_{j}, \gamma_{0}\left(w_{j}\right)\right)$, and summing once more from $j=1$ to $m$, we obtain

$$
\begin{aligned}
& \left(A_{1}\left(\left(u_{m}(r), \gamma_{0}\left(u_{m}(r)\right)\right)\right),\left(u_{m}^{\prime}(r), \gamma_{0}\left(u_{m}^{\prime}(r)\right)\right)\right)_{H}+\left|A_{1}\left(\left(u_{m}(r), \gamma_{0}\left(u_{m}(r)\right)\right)\right)\right|_{H}^{2} \\
+ & \left(f\left(u_{m}(r)\right), \sum_{j=1}^{m} \lambda_{j} \delta_{m j}(r) w_{j}\right)_{\Omega}+\left(g\left(\gamma_{0}\left(u_{m}(r)\right)\right), \sum_{j=1}^{m} \lambda_{j} \delta_{m j}(r) \gamma_{0}\left(w_{j}\right)\right)_{\partial \Omega} \\
= & \left(h(r), \sum_{j=1}^{m} \lambda_{j} \delta_{m j}(r) w_{j}\right)_{\Omega}+\left(\rho(r), \sum_{j=1}^{m} \lambda_{j} \delta_{m j}(r) \gamma_{0}\left(w_{j}\right)\right)_{\partial \Omega}, \quad \text { a.e. } r>\tau .
\end{aligned}
$$

It follows from the properties of $f,(14)$, and integrating by parts, that

$$
\begin{aligned}
& -\left(f\left(u_{m}(r)\right), \sum_{j=1}^{m} \lambda_{j} \delta_{m j} w_{j}\right)_{\Omega} \\
= & -\int_{\Omega} f\left(u_{m}(x, r)\right)\left(-\Delta u_{m}(x, r)+\kappa u_{m}(x, r)\right) d x \\
= & -\int_{\Omega} f^{\prime}\left(u_{m}(x, r)\right)\left|\nabla u_{m}(x, r)\right|^{2} d x+\int_{\partial \Omega} f\left(\gamma_{0}\left(u_{m}(x, r)\right)\right) \frac{\partial u_{m}}{\partial \vec{n}} d \sigma(x)-\kappa \int_{\Omega} f\left(u_{m}(x, r)\right) u_{m}(x, r) d x \\
\leq & l\left|\nabla u_{m}(r)\right|_{\Omega}^{2}+\int_{\partial \Omega} f\left(\gamma_{0}\left(u_{m}(x, r)\right)\right) \frac{\partial u_{m}}{\partial \vec{n}} d \sigma(x)-\kappa \alpha_{1}\left|u_{m}(r)\right|_{p, \Omega}^{p}+\kappa \beta|\Omega| \quad \forall r \geq \tau .
\end{aligned}
$$

On the other hand, we have

$$
-\left(g\left(\gamma_{0}\left(u_{m}(r)\right)\right), \sum_{j=1}^{m} \lambda_{j} \delta_{m j}(r) \gamma_{0}\left(w_{j}\right)\right)_{\partial \Omega}=-\int_{\partial \Omega} g\left(\gamma_{0}\left(u_{m}(x, r)\right)\right) \frac{\partial u_{m}}{\partial \vec{n}} d \sigma(x) \quad \forall r \geq \tau,
$$

whence, jointly with the last inequality,

$$
\begin{aligned}
&-\left(f\left(u_{m}(r)\right), \sum_{j=1}^{m} \lambda_{j} \delta_{m j}(r) w_{j}\right)_{\Omega}-\left(g\left(\gamma_{0}\left(u_{m}(r)\right)\right), \sum_{j=1}^{m} \lambda_{j} \delta_{m j}(r) \gamma_{0}\left(w_{j}\right)\right)_{\partial \Omega} \\
& \leq l\left|\nabla u_{m}(r)\right|_{\Omega}^{2}-\kappa \alpha_{1}\left|u_{m}(r)\right|_{p, \Omega}^{p}+\kappa \beta|\Omega|+\int_{\partial \Omega}\left(f\left(\gamma_{0}\left(u_{m}(x, r)\right)\right)-g\left(\gamma_{0}\left(u_{m}(x, r)\right)\right)\right) \frac{\partial u_{m}}{\partial \vec{n}} d \sigma(x)
\end{aligned}
$$

for all $r \geq \tau$. 
Using (13) and Cauchy-Schwartz and Young inequalities, we obtain

$$
\begin{aligned}
& -\left(f\left(u_{m}(r)\right), \sum_{j=1}^{m} \lambda_{j} \delta_{m j}(r) w_{j}\right)_{\Omega}-\left(g\left(\gamma_{0}\left(u_{m}(r)\right)\right), \sum_{j=1}^{m} \lambda_{j} \delta_{m j}(r) \gamma_{0}\left(w_{j}\right)\right)_{\partial \Omega} \\
\leq \quad & l\left|\nabla u_{m}(r)\right|_{\Omega}^{2}-\kappa \alpha_{1}\left|u_{m}(r)\right|_{p, \Omega}^{p}+\kappa \beta|\Omega|+2^{1 / 2} c_{f g}\left(|\partial \Omega|^{1 / 2}+\left|\gamma_{0}\left(u_{m}(r)\right)\right|_{\partial \Omega}\right)\left|\frac{\partial u_{m}}{\partial \vec{n}}\right|_{\partial \Omega} \\
\leq \quad & l\left|\nabla u_{m}(r)\right|_{\Omega}^{2}-\kappa \alpha_{1}\left|u_{m}(r)\right|_{p, \Omega}^{p}+\kappa \beta|\Omega|+4 c_{f g}^{2}\left(|\partial \Omega|+\left|\gamma_{0}\left(u_{m}(r)\right)\right|_{\partial \Omega}^{2}\right) \\
& +\frac{1}{4}\left|A_{1}\left(\left(u_{m}(r), \gamma_{0}\left(u_{m}(r)\right)\right)\right)\right|_{H}^{2} \quad \forall r \geq \tau .
\end{aligned}
$$

On the other hand, we have

$$
\begin{aligned}
\left(h(r), \sum_{j=1}^{m} \lambda_{j} \delta_{m j}(r) w_{j}\right)_{\Omega} & \leq 2|h(r)|_{\Omega}^{2}+\frac{1}{8}\left|A_{1}\left(\left(u_{m}(r), \gamma_{0}\left(u_{m}(r)\right)\right)\right)\right|_{H}^{2}, \quad \text { a.e. } r>\tau \\
\left(\rho(r), \sum_{j=1}^{m} \lambda_{j} \delta_{m j}(r) \gamma_{0}\left(w_{j}\right)\right)_{\partial \Omega} & \leq 2|\rho(r)|_{\partial \Omega}^{2}+\frac{1}{8}\left|A_{1}\left(\left(u_{m}(r), \gamma_{0}\left(u_{m}(r)\right)\right)\right)\right|_{H}^{2}, \quad \text { a.e. } r>\tau .
\end{aligned}
$$

Using the above inequalities in (16), in particular, we obtain

$$
\begin{aligned}
\left|A_{1}\left(\left(u_{m}(r), \gamma_{0}\left(u_{m}(r)\right)\right)\right)\right|_{H}^{2} \leq & 4\left|\left(u_{m}^{\prime}(r), \gamma_{0}\left(u_{m}^{\prime}(r)\right)\right)\right|_{H}^{2}+4 l\left|\nabla u_{m}(r)\right|_{\Omega}^{2}+4 \kappa \beta|\Omega| \\
& +16 c_{f g}^{2}\left(|\partial \Omega|+\left|\gamma_{0}\left(u_{m}(r)\right)\right|_{\partial \Omega}^{2}\right)+8\left(|h(r)|_{\Omega}^{2}+|\rho(r)|_{\partial \Omega}^{2}\right)
\end{aligned}
$$

a.e. $r \geq \tau$.

Finally, integrating the last inequality between $\tau$ and $T$, taking into account Lemma 3 and that $\left\{\left(u_{m}^{\prime}, \gamma_{0}\left(u_{m}^{\prime}\right)\right)\right\}$ is bounded in $L^{2}(\tau, T ; H)$, we obtain that $\left\{\left(u_{m}, \gamma_{0}\left(u_{m}\right)\right)\right\}$ is bounded in $L^{2}\left(\tau, T ; D\left(A_{1}\right)\right)$. Step 2 and the proof of the theorem are concluded.

As a consequence of the last result and Theorem 5 in [4], we can now establish the following result.

Theorem 5 Under the assumptions in Theorem 4, for any initial condition $\left(u_{\tau}, \psi_{\tau}\right) \in H$, the weak solution to (1)-(4) satisfies

$$
\begin{gathered}
(u, \psi) \in C\left((\tau, T] ; V_{1}\right) \cap L^{2}\left(\tau+\varepsilon, T ; D\left(A_{1}\right)\right) \quad \forall T>\tau+\varepsilon>\tau, \\
\left(u^{\prime}, \psi^{\prime}\right) \in L^{2}(\tau+\varepsilon, T ; H) \quad \forall T>\tau+\varepsilon>\tau
\end{gathered}
$$

\section{$3 D\left(A_{1}\right)$-boundedness of invariants sets}

We start recalling a result (see [24, Lemma 2.1]) which is necessary below.

Lemma 6 Let $X, Y$ be Banach spaces such that $X$ is reflexive, and the inclusion $X \subset Y$ is continuous. Assume that $\left\{u_{m}\right\}$ is a bounded sequence in $L^{\infty}\left(t_{0}, T ; X\right)$ such that $u_{m} \rightarrow u$ weakly in $L^{q}\left(t_{0}, T ; X\right)$ for some $q \in[1, \infty)$ and $u \in C\left(\left[t_{0}, T\right] ; Y\right)$.

Then, $u(t) \in X$ for all $t \in\left[t_{0}, T\right]$ and

$$
\|u(t)\|_{X} \leq \liminf _{m \rightarrow \infty}\left\|u_{m}\right\|_{L^{\infty}\left(t_{0}, T ; X\right)} \quad \forall t \in\left[t_{0}, T\right] .
$$


We first prove the following result.

Proposition 7 Under the assumptions in Theorem 4, for any bounded set $B \subset H, \tau \in \mathbb{R}$, $\varepsilon>0$ and $t>\tau+\varepsilon$, the set $\left\{\left(u_{m}\left(r ; \tau, u_{\tau}, \psi_{\tau}\right), \gamma_{0}\left(u_{m}\left(r ; \tau, u_{\tau}, \psi_{\tau}\right)\right)\right): r \in[\tau+\varepsilon, t],\left(u_{\tau}, \psi_{\tau}\right) \in\right.$ $B, m \geq 1\}$ is a bounded subset of $V_{1} \cap\left(L^{p}(\Omega) \times L^{p}(\partial \Omega)\right)$.

Proof. Let us fix a bounded set $B \subset H, \tau \in \mathbb{R}, \varepsilon>0, t>\tau+\varepsilon$, and $\left(u_{\tau}, \psi_{\tau}\right) \in B$.

We will consider $P_{m}\left(\left(u_{\tau}, \psi_{\tau}\right)\right):=\sum_{i=1}^{m}\left(\left(u_{\tau}, \psi_{\tau}\right),\left(w_{i}, \gamma_{0}\left(w_{i}\right)\right)\right)_{H}\left(w_{i}, \gamma_{0}\left(w_{i}\right)\right)$ as initial data $\left(u_{\tau_{m}}, \gamma_{0}\left(u_{\tau_{m}}\right)\right)$, which generates a sequence that converges to $\left(u_{\tau}, \psi_{\tau}\right)$ in $H$. Now, for the corresponding solutions to the Galerkin system, integrating (12) between $\tau$ and $r$, we have

$$
\begin{aligned}
& \left|\left(u_{m}(r), \gamma_{0}\left(u_{m}(r)\right)\right)\right|_{H}^{2}+\frac{\min \{1, \kappa\}}{1+\left\|\gamma_{0}\right\|^{2}} \int_{\tau}^{r}\left\|\left(u_{m}(s), \gamma_{0}\left(u_{m}(s)\right)\right)\right\|_{V_{1}}^{2} d s \\
& +2 \alpha_{1} \int_{\tau}^{r}\left|u_{m}(s)\right|_{p, \Omega}^{p} d s+2 \alpha_{1} \int_{\tau}^{r}\left|\gamma_{0}\left(u_{m}(s)\right)\right|_{p, \partial \Omega}^{p} d s \\
\leq & 2 \beta(t-\tau)(|\Omega|+|\partial \Omega|)+\frac{2}{\kappa} \int_{\tau}^{t}|h(s)|_{\Omega}^{2} d s+\frac{\left\|\gamma_{0}\right\|^{2}}{\min \{1, \kappa / 2\}} \int_{\tau}^{t}|\rho(s)|_{\partial \Omega}^{2} d s+\left|\left(u_{\tau}, \psi_{\tau}\right)\right|_{H}^{2}
\end{aligned}
$$

for all $r \in[\tau, t]$, for all $m \geq 1$.

Integrating now (15) between $s$ and $r$ with $\tau \leq s \leq r \leq t$, we obtain

$$
\begin{aligned}
& \int_{s}^{r}\left|\left(u_{m}^{\prime}(\theta), \gamma_{0}\left(u_{m}^{\prime}(\theta)\right)\right)\right|_{H}^{2} d \theta+\left\langle A_{1}\left(\left(u_{m}(r), \gamma_{0}\left(u_{m}(r)\right)\right)\right),\left(u_{m}(r), \gamma_{0}\left(u_{m}(r)\right)\right)\right\rangle \\
\leq & \left\langle A_{1}\left(\left(u_{m}(s), \gamma_{0}\left(u_{m}(s)\right)\right)\right),\left(u_{m}(s), \gamma_{0}\left(u_{m}(s)\right)\right)\right\rangle+\int_{\tau}^{t}\left(|h(\theta)|_{\Omega}^{2}+|\rho(\theta)|_{\partial \Omega}^{2}\right) d \theta \\
& +2 \int_{\Omega}\left(\mathcal{F}\left(u_{m}(x, s)\right)-\mathcal{F}\left(u_{m}(x, r)\right)\right) d x+2 \int_{\partial \Omega}\left(\mathcal{G}\left(\gamma_{0}\left(u_{m}(x, s)\right)\right)-\mathcal{G}\left(\gamma_{0}\left(u_{m}(x, r)\right)\right)\right) d \sigma(x),
\end{aligned}
$$

which, jointly with the inequality (16) of [4], (5) and (6), yields that

$$
\begin{aligned}
& \int_{s}^{r}\left|\left(u_{m}^{\prime}(\theta), \gamma_{0}\left(u_{m}^{\prime}(\theta)\right)\right)\right|_{H}^{2} d \theta+\frac{\min \{1, \kappa\}}{1+\left\|\gamma_{0}\right\|^{2}}\left\|\left(u_{m}(r), \gamma_{0}\left(u_{m}(r)\right)\right)\right\|_{V_{1}}^{2} \\
& +2 \widetilde{\alpha}_{1}\left|u_{m}(r)\right|_{p, \Omega}^{p}+2 \widetilde{\alpha}_{1}\left|\gamma_{0}\left(u_{m}(r)\right)\right|_{p, \partial \Omega}^{p} \\
\leq & \max \{1, \kappa\}\left\|\left(u_{m}(s), \gamma_{0}\left(u_{m}(s)\right)\right)\right\|_{V_{1}}^{2}+\int_{\tau}^{t}\left(|h(\theta)|_{\Omega}^{2}+|\rho(\theta)|_{\partial \Omega}^{2}\right) d \theta \\
& +2 \widetilde{\alpha}_{2}\left|u_{m}(s)\right|_{p, \Omega}^{p}+2 \widetilde{\alpha}_{2}\left|\gamma_{0}\left(u_{m}(s)\right)\right|_{p, \partial \Omega}^{p}+4 \widetilde{\beta}(|\Omega|+|\partial \Omega|)
\end{aligned}
$$

for any $s$ and $r$ with $\tau \leq s \leq r \leq t$.

Integrating this last inequality with respect to $s$ from $\tau$ to $r$, in particular, we obtain

$$
\begin{aligned}
& (r-\tau)\left(\frac{\min \{1, \kappa\}}{1+\left\|\gamma_{0}\right\|^{2}}\left\|\left(u_{m}(r), \gamma_{0}\left(u_{m}(r)\right)\right)\right\|_{V_{1}}^{2}+2 \widetilde{\alpha}_{1}\left(\left|u_{m}(r)\right|_{p, \Omega}^{p}+\left|\gamma_{0}\left(u_{m}(r)\right)\right|_{p, \partial \Omega}^{p}\right)\right) \\
\leq & \max \{1, \kappa\} \int_{\tau}^{r}\left\|\left(u_{m}(s), \gamma_{0}\left(u_{m}(s)\right)\right)\right\|_{V_{1}}^{2} d s+(t-\tau) \int_{\tau}^{t}\left(|h(s)|_{\Omega}^{2}+|\rho(s)|_{\partial \Omega}^{2}\right) d s \\
& +2 \widetilde{\alpha}_{2} \int_{\tau}^{r}\left(\left|u_{m}(s)\right|_{p, \Omega}^{p}+\left|\gamma_{0}\left(u_{m}(s)\right)\right|_{p, \partial \Omega}^{p}\right) d s+(t-\tau) 4 \widetilde{\beta}(|\Omega|+|\partial \Omega|)
\end{aligned}
$$

for all $r \in[\tau, t]$, and for any $m \geq 1$.

From this inequality and (18), the result follows. 
Corollary 8 Under the assumptions in Theorem 4, for any bounded set $B \subset H, \tau \in \mathbb{R}, \varepsilon>0$, and $t>\tau+\varepsilon$, the set $\cup_{r \in[\tau+\varepsilon, t]} U(r, \tau) B$ is a bounded subset of $V_{1} \cap\left(L^{p}(\Omega) \times L^{p}(\partial \Omega)\right)$.

Proof. This is a straightforward consequence of Lemma 6, Proposition 7, the fact that $\left(u\left(\cdot ; \tau, u_{\tau}, \psi_{\tau}\right), \gamma_{0}\left(u\left(\cdot ; \tau, u_{\tau}, \psi_{\tau}\right)\right)\right) \in C\left([\tau+\varepsilon, t] ; V_{1}\right)$ and the fact that the Galerkin sequence $\left\{\left(u_{m}\left(\cdot ; \tau, u_{\tau}, \psi_{\tau}\right), \gamma_{0}\left(u_{m}\left(\cdot ; \tau, u_{\tau}, \psi_{\tau}\right)\right)\right)\right\}$ converges weakly, thanks to monotonicity arguments (cf. [32, Chapter 2] and [4, Theorem 5]), to $\left(u\left(\cdot ; \tau, u_{\tau}, \psi_{\tau}\right), \gamma_{0}\left(u\left(\cdot ; \tau, u_{\tau}, \psi_{\tau}\right)\right)\right)$, the unique solution to (1)-(4), in $L^{2}\left(\tau, T ; V_{1}\right)$ and $L^{p}\left(\tau, T ; L^{p}(\Omega) \times L^{p}(\partial \Omega)\right)$.

Proposition 9 Suppose that, in addition to the assumptions in Theorem 4, it holds that $h \in$ $W_{\text {loc }}^{1,2}\left(\mathbb{R} ; L^{2}(\Omega)\right)$ and $\rho \in W_{\text {loc }}^{1,2}\left(\mathbb{R} ; L^{2}(\partial \Omega)\right)$.

Then, for any bounded set $B \subset H, \tau \in \mathbb{R}, \varepsilon>0$, and $t>\tau+\varepsilon$, the set

$$
\left\{\left(u_{m}\left(r ; \tau, u_{\tau}, \psi_{\tau}\right), \gamma_{0}\left(u_{m}\left(r ; \tau, u_{\tau}, \psi_{\tau}\right)\right)\right): r \in[\tau+\varepsilon, t],\left(u_{\tau}, \psi_{\tau}\right) \in B, m \geq 1\right\}
$$

is a bounded subset of $D\left(A_{1}\right)$.

Proof. Let us fix a bounded set $B \subset H, \tau \in \mathbb{R}, \varepsilon>0, t>\tau+\varepsilon$, and $\left(u_{\tau}, \psi_{\tau}\right) \in B$. As we are assuming that $f, g \in \mathcal{C}^{1}(\mathbb{R}), h \in W_{\text {loc }}^{1,2}\left(\mathbb{R} ; L^{2}(\Omega)\right)$ and $\rho \in W_{\text {loc }}^{1,2}\left(\mathbb{R} ; L^{2}(\partial \Omega)\right)$, we can differentiate with respect to time in (10), and then, multiplying by $\delta_{m j}^{\prime}$, and summing from $j=1$ to $m$, we obtain

$$
\begin{aligned}
& \frac{1}{2} \frac{d}{d r}\left|\left(u_{m}^{\prime}(r), \gamma_{0}\left(u_{m}^{\prime}(r)\right)\right)\right|_{H}^{2}+\left\langle A_{1}\left(\left(u_{m}^{\prime}(r), \gamma_{0}\left(u_{m}^{\prime}(r)\right)\right)\right),\left(u_{m}^{\prime}(r), \gamma_{0}\left(u_{m}^{\prime}(r)\right)\right)\right\rangle \\
= & -\left(f^{\prime}\left(u_{m}(r)\right) u_{m}^{\prime}(r), u_{m}^{\prime}(r)\right)_{\Omega}-\left(g^{\prime}\left(\gamma_{0}\left(u_{m}(r)\right)\right) \gamma_{0}\left(u_{m}^{\prime}(r)\right), \gamma_{0}\left(u_{m}^{\prime}(r)\right)\right)_{\partial \Omega} \\
& +\left(h^{\prime}(r), u_{m}^{\prime}(r)\right)_{\Omega}+\left(\rho^{\prime}(r), \gamma_{0}\left(u_{m}^{\prime}(r)\right)\right)_{\partial \Omega}, \quad \text { a.e. } r>\tau .
\end{aligned}
$$

Then, using (14), we have

$$
\begin{aligned}
& \frac{d}{d r}\left|\left(u_{m}^{\prime}(r), \gamma_{0}\left(u_{m}^{\prime}(r)\right)\right)\right|_{H}^{2} \\
\leq & 2 l\left|u_{m}^{\prime}(r)\right|_{\Omega}^{2}+2 l\left|\gamma_{0}\left(u_{m}^{\prime}(r)\right)\right|_{\partial \Omega}^{2}+\left|h^{\prime}(r)\right|_{\Omega}^{2}+\left|u_{m}^{\prime}(r)\right|_{\Omega}^{2}+\left|\rho^{\prime}(r)\right|_{\partial \Omega}^{2}+\left|\gamma_{0}\left(u_{m}^{\prime}(r)\right)\right|_{\partial \Omega}^{2}, \quad \text { a.e. } r>\tau .
\end{aligned}
$$

In particular, integrating in the last inequality,

$$
\begin{aligned}
\left|\left(u_{m}^{\prime}(r), \gamma_{0}\left(u_{m}^{\prime}(r)\right)\right)\right|_{H}^{2} \leq & \left|\left(u_{m}^{\prime}(s), \gamma_{0}\left(u_{m}^{\prime}(s)\right)\right)\right|_{H}^{2}+(2 l+1) \int_{\tau+\varepsilon / 2}^{t}\left(\left|u_{m}^{\prime}(\theta)\right|_{\Omega}^{2}+\left|\gamma_{0}\left(u_{m}^{\prime}(\theta)\right)\right|_{\partial \Omega}^{2}\right) d \theta \\
& +\int_{\tau+\varepsilon / 2}^{t}\left(\left|h^{\prime}(\theta)\right|_{\Omega}^{2}+\left|\rho^{\prime}(\theta)\right|_{\partial \Omega}^{2}\right) d \theta
\end{aligned}
$$

for all $\tau+\varepsilon / 2 \leq s \leq r \leq t$.

Now, integrating with respect to $s$ between $\tau+\varepsilon / 2$ and $r$, we have

$$
\begin{aligned}
& (r-\tau-\varepsilon / 2)\left|\left(u_{m}^{\prime}(r), \gamma_{0}\left(u_{m}^{\prime}(r)\right)\right)\right|_{H}^{2} \\
\leq & {[(2 l+1)(t-\tau-\varepsilon / 2)+1] \int_{\tau+\varepsilon / 2}^{t}\left|\left(u_{m}^{\prime}(\theta), \gamma_{0}\left(u_{m}^{\prime}(\theta)\right)\right)\right|_{H}^{2} d \theta } \\
& +(r-\tau-\varepsilon / 2) \int_{\tau+\varepsilon / 2}^{t}\left(\left|h^{\prime}(\theta)\right|_{\Omega}^{2}+\left|\rho^{\prime}(\theta)\right|_{\partial \Omega}^{2}\right) d \theta
\end{aligned}
$$


for all $\tau+\varepsilon / 2 \leq r \leq t$. In particular,

$$
\begin{aligned}
& \left|\left(u_{m}^{\prime}(r), \gamma_{0}\left(u_{m}^{\prime}(r)\right)\right)\right|_{H}^{2} \\
\leq & 2 \varepsilon^{-1}((2 l+1)(t-\tau-\varepsilon / 2)+1) \int_{\tau+\varepsilon / 2}^{t}\left|\left(u_{m}^{\prime}(\theta), \gamma_{0}\left(u_{m}^{\prime}(\theta)\right)\right)\right|_{H}^{2} d \theta+\int_{\tau+\varepsilon / 2}^{t}\left(\left|h^{\prime}(\theta)\right|_{\Omega}^{2}+\left|\rho^{\prime}(\theta)\right|_{\partial \Omega}^{2}\right) d \theta
\end{aligned}
$$

for all $r \in[\tau+\varepsilon, t]$.

Finally, observe that by (19)

$$
\begin{aligned}
& \int_{\tau+\varepsilon / 2}^{t}\left|\left(u_{m}^{\prime}(\theta), \gamma_{0}\left(u_{m}^{\prime}(\theta)\right)\right)\right|_{H}^{2} d \theta \\
\leq & \max \{1, k\}\left\|\left(u_{m}(\tau+\varepsilon / 2), \gamma_{0}\left(u_{m}(\tau+\varepsilon / 2)\right)\right)\right\|_{V_{1}}^{2}+\int_{\tau}^{t}\left(|h(\theta)|_{\Omega}^{2}+|\rho(\theta)|_{\partial \Omega}^{2}\right) d \theta \\
& +2 \widetilde{\alpha}_{2}\left|u_{m}(\tau+\varepsilon / 2)\right|_{p, \Omega}^{p}+2 \widetilde{\alpha}_{2}\left|\gamma_{0}\left(u_{m}(\tau+\varepsilon / 2)\right)\right|_{p, \partial \Omega}^{p}+4 \widetilde{\beta}(|\Omega|+|\partial \Omega|) .
\end{aligned}
$$

Taking into account that, in particular, $h \in C\left([\tau, t] ; L^{2}(\Omega)\right)$ and $\rho \in C\left([\tau, t] ; L^{2}(\partial \Omega)\right)$, the result is a direct consequence of Proposition 7 and estimates (17), (21) and (22).

Corollary 10 Under the assumptions of Proposition 9, for any bounded set $B \subset H, \tau \in \mathbb{R}$, $\varepsilon>0$, and $t>\tau+\varepsilon$, the set $\cup_{r \in[\tau+\varepsilon, t]} U(r, \tau) B$ is a bounded subset of $D\left(A_{1}\right)$.

Proof. This result is a straightforward consequence of Lemma 6, Propositions 7 and 9, the facts that, using uniqueness and monotonicity arguments (cf. [32, Chapter 2] and [4, Theorem $5]),\left\{\left(u_{m}\left(\cdot ; \tau, u_{\tau}, \psi_{\tau}\right), \gamma_{0}\left(u_{m}\left(\cdot ; \tau, u_{\tau}, \psi_{\tau}\right)\right)\right)\right\}$ converges weakly to the unique solution to (1)-(4) $\left(u\left(\cdot ; \tau, u_{\tau}, \psi_{\tau}\right), \gamma_{0}\left(u\left(\cdot ; \tau, u_{\tau}, \psi_{\tau}\right)\right)\right)$ in $L^{2}\left(\tau, T ; D\left(A_{1}\right)\right)$ and that $\left(u\left(\cdot ; \tau, u_{\tau}, \psi_{\tau}\right), \gamma_{0}\left(u\left(\cdot ; \tau, u_{\tau}, \psi_{\tau}\right)\right)\right)$ $\in C\left([\tau+\varepsilon, t] ; V_{1}\right)$.

As a direct consequence of the above results, we can now establish our main result.

Proposition 11 Under the assumptions in Proposition 9, if $\mathcal{A}=\{\mathcal{A}(t): t \in \mathbb{R}\}$ is a family of bounded subsets of $H$, such that $U(t, \tau) \mathcal{A}(\tau)=\mathcal{A}(t)$ for any $\tau \leq t$, then for any $T_{1}<T_{2}$, the set $\cup_{t \in\left[T_{1}, T_{2}\right]} \mathcal{A}(t)$ is a bounded subset of $D\left(A_{1}\right) \cap V_{1} \cap\left(L^{p}(\Omega) \times L^{p}(\partial \Omega)\right)$.

In particular, we have the following result for pullback attractors.

Corollary 12 Under the assumptions in Proposition 9, if $\mathcal{A}=\{\mathcal{A}(t): t \in \mathbb{R}\}$ is a pullback attractor for the process $U$, then for any $T_{1}<T_{2}$, the set $\cup_{t \in\left[T_{1}, T_{2}\right]} \mathcal{A}(t)$ is a bounded subset of $D\left(A_{1}\right) \cap V_{1} \cap\left(L^{p}(\Omega) \times L^{p}(\partial \Omega)\right)$.

\section{An exponential growth condition for pullback attrac- tors}

The aim of this section is to continue with the analysis of the model in the sense of proving that the family $\mathcal{A}_{\mathcal{D}_{\mu}^{H}}$, for short denoted simply $\mathcal{A}$ (whose existence was guaranteed in [4, Theorem $23]$ ), satisfies also an exponential growth condition on the space $V_{1} \cap\left(L^{p}(\Omega) \times L^{p}(\partial \Omega)\right)$, and finally in $D\left(A_{1}\right)$, provided some additional assumptions are fulfilled. 
Theorem 13 Under the assumptions in Theorem 4, if, moreover, condition (7) holds, then $\mathcal{A}$ satisfies

$$
\lim _{\tau \rightarrow-\infty} e^{\mu \tau}\left(\sup _{(v, \phi) \in \mathcal{A}(\tau)}\|(v, \phi)\|_{V_{1}}^{2}+\sup _{(v, \phi) \in \mathcal{A}(\tau)}\left(|v|_{p, \Omega}^{p}+|\phi|_{p, \partial \Omega}^{p}\right)\right)=0 .
$$

Proof. From the inequality (18), for any $t \geq \tau$, we have

$$
\begin{aligned}
& \left|\left(u_{m}(r), \gamma_{0}\left(u_{m}(r)\right)\right)\right|_{H}^{2}+\int_{\tau}^{r}\left(\left\|\left(u_{m}(s), \gamma_{0}\left(u_{m}(s)\right)\right)\right\|_{V_{1}}^{2}+\left|u_{m}(s)\right|_{p, \Omega}^{p}+\left|\gamma_{0}\left(u_{m}(s)\right)\right|_{p, \partial \Omega}^{p}\right) d s \\
\leq & C_{1}\left(\int_{\tau}^{t}\left(|h(s)|_{\Omega}^{2}+|\rho(s)|_{\partial \Omega}^{2}\right) d s+t-\tau+\left|\left(u_{\tau}, \psi_{\tau}\right)\right|_{H}^{2}\right)
\end{aligned}
$$

for all $r \in[\tau, t]$, and for all $m \geq 1$, where

$$
C_{1}=\frac{\max \left\{2 \beta(|\Omega|+|\partial \Omega|), 2 \kappa^{-1},\left\|\gamma_{0}\right\|^{2} / \min \{1, \kappa / 2\}, 1\right\}}{\min \left\{1, \min \{1, \kappa\} /\left(1+\left\|\gamma_{0}\right\|^{2}\right), 2 \alpha_{1}\right\}} .
$$

On other hand, from (20) we have

$$
\begin{aligned}
& (r-\tau)\left(\left\|\left(u_{m}(r), \gamma_{0}\left(u_{m}(r)\right)\right)\right\|_{V_{1}}^{2}+\left|u_{m}(r)\right|_{p, \Omega}^{p}+\left|\gamma_{0}\left(u_{m}(r)\right)\right|_{p, \partial \Omega}^{p}\right) \\
\leq & C_{2} \int_{\tau}^{r}\left(\left\|\left(u_{m}(s), \gamma_{0}\left(u_{m}(s)\right)\right)\right\|_{V_{1}}^{2}+\left|u_{m}(s)\right|_{p, \Omega}^{p}+\left|\gamma_{0}\left(u_{m}(s)\right)\right|_{p, \partial \Omega}^{p}\right) d s \\
& +C_{2}(t-\tau) \int_{\tau}^{t}\left(|h(s)|_{\Omega}^{2}+|\rho(s)|_{\partial \Omega}^{2}\right) d s+C_{2}(t-\tau)
\end{aligned}
$$

for any $t \geq \tau$, all $r \in[\tau, t]$, and all $m \geq 1$, where

$$
C_{2}=\frac{\max \left\{1, \kappa, 2 \widetilde{\alpha}_{2}, 4 \widetilde{\beta}(|\Omega|+|\partial \Omega|)\right\}}{\min \left\{\min \{1, \kappa\} /\left(1+\left\|\gamma_{0}\right\|^{2}\right), 2 \widetilde{\alpha}_{1}\right\}} .
$$

From (24) and the above inequality we obtain that

$$
\begin{aligned}
& (r-\tau)\left(\left\|\left(u_{m}(r), \gamma_{0}\left(u_{m}(r)\right)\right)\right\|_{V_{1}}^{2}+\left|u_{m}(r)\right|_{p, \Omega}^{p}+\left|\gamma_{0}\left(u_{m}(r)\right)\right|_{p, \partial \Omega}^{p}\right) \\
\leq & C_{2}\left(C_{1}+t-\tau\right) \int_{\tau}^{t}\left(|h(s)|_{\Omega}^{2}+|\rho(s)|_{\partial \Omega}^{2}\right) d s+C_{2}(t-\tau)\left(1+C_{1}\right)+C_{2} C_{1}\left|\left(u_{\tau}, \psi_{\tau}\right)\right|_{H}^{2}
\end{aligned}
$$

for any $t \geq \tau$, all $r \in[\tau, t]$, and all $m \geq 1$.

In particular from above we deduce

$$
\begin{aligned}
& \left\|\left(u_{m}(r), \gamma_{0}\left(u_{m}(r)\right)\right)\right\|_{V_{1}}^{2}+\left|u_{m}(r)\right|_{p, \Omega}^{p}+\left|\gamma_{0}\left(u_{m}(r)\right)\right|_{p, \partial \Omega}^{p} \\
\leq & C_{3}\left(\left|\left(u_{\tau}, \psi_{\tau}\right)\right|_{H}^{2}+\int_{\tau}^{\tau+3}\left(|h(s)|_{\Omega}^{2}+|\rho(s)|_{\partial \Omega}^{2}\right) d s+1\right)
\end{aligned}
$$

for all $r \in[\tau+1, \tau+3]$, and any $m \geq 1$, where $C_{3}=3 C_{2}\left(C_{1}+1\right)$.

As $\left(u\left(\cdot ; \tau, u_{\tau}, \psi_{\tau}\right), \gamma_{0}\left(u\left(\cdot ; \tau, u_{\tau}, \psi_{\tau}\right)\right)\right) \in C\left([\tau+1, t] ; V_{1}\right)$ for all $t>\tau+1$ and since it holds that $\left\{\left(u_{m}\left(\cdot ; \tau, u_{\tau}, \psi_{\tau}\right), \gamma_{0}\left(u_{m}\left(\cdot ; \tau, u_{\tau}, \psi_{\tau}\right)\right)\right)\right\}$ converges weakly to $\left(u\left(\cdot ; \tau, u_{\tau}, \psi_{\tau}\right), \gamma_{0}\left(u\left(\cdot ; \tau, u_{\tau}, \psi_{\tau}\right)\right)\right)$ 
in $L^{2}\left(\tau, t ; V_{1}\right)$ and in $L^{p}\left(\tau, t ; L^{p}(\Omega) \times L^{p}(\partial \Omega)\right)$ for all $t>\tau$ using Lemma 3, from (25) and Lemma 6 , in particular, we obtain

$$
\begin{aligned}
& \left\|\left(u(\tau+1), \gamma_{0}(u(\tau+1))\right)\right\|_{V_{1}}^{2}+|u(\tau+1)|_{p, \Omega}^{p}+\left|\gamma_{0}(u(\tau+1))\right|_{p, \partial \Omega}^{p} \\
\leq & C_{3}\left(\left|\left(u_{\tau}, \psi_{\tau}\right)\right|_{H}^{2}+\int_{\tau}^{\tau+3}\left(|h(s)|_{\Omega}^{2}+|\rho(s)|_{\partial \Omega}^{2}\right) d s+1\right) .
\end{aligned}
$$

Then, multiplying this inequality by $e^{\mu(\tau+1)}$ we have

$$
\begin{aligned}
& e^{\mu(\tau+1)}\left(\left\|\left(u(\tau+1), \gamma_{0}(u(\tau+1))\right)\right\|_{V_{1}}^{2}+|u(\tau+1)|_{p, \Omega}^{p}+\left|\gamma_{0}(u(\tau+1))\right|_{p, \partial \Omega}^{p}\right) \\
\leq & C_{3} e^{\mu}\left(e^{\mu \tau}\left|\left(u_{\tau}, \psi_{\tau}\right)\right|_{H}^{2}+\int_{\tau}^{\tau+3} e^{\mu s}\left(|h(s)|_{\Omega}^{2} d s+|\rho(s)|_{\partial \Omega}^{2}\right) d s+e^{\mu \tau}\right)
\end{aligned}
$$

for all $\tau \in \mathbb{R}$, and all $\left(u_{\tau}, \psi_{\tau}\right) \in H$.

As $\mathcal{A}(\tau+1)=U(\tau+1, \tau) \mathcal{A}(\tau)$, it follows from above that

$$
\begin{aligned}
& e^{\mu(\tau+1)}\left(\|(v, \phi)\|_{V_{1}}^{2}+|v|_{p, \Omega}^{p}+|\phi|_{p, \partial \Omega}^{p}\right) \\
\leq & C_{3} e^{\mu}\left(e^{\mu \tau} \sup _{(w, \psi) \in \mathcal{A}(\tau)}|(w, \psi)|_{H}^{2}+\int_{\tau}^{\tau+3} e^{\mu s}\left(|h(s)|_{\Omega}^{2}+|\rho(s)|_{\partial \Omega}^{2}\right) d s+e^{\mu \tau}\right)
\end{aligned}
$$

for all $(v, \phi) \in \mathcal{A}(\tau+1)$, and any $\tau \in \mathbb{R}$.

Taking into account (7) and (8), from above we obtain (23).

Theorem 14 Under the assumptions in Proposition 9, if, moreover, condition (7) holds, and $h$ and $\rho$ satisfy

$$
\lim _{\tau \rightarrow-\infty} e^{\mu \tau} \int_{\tau}^{\tau+1}\left(\left|h^{\prime}(s)\right|_{\Omega}^{2}+\left|\rho^{\prime}(s)\right|_{\partial \Omega}^{2}\right) d s=0,
$$

and

$$
\lim _{\tau \rightarrow-\infty} e^{\mu \tau}\left(|h(\tau)|_{\Omega}^{2}+|\rho(\tau)|_{\partial \Omega}^{2}\right) d s=0
$$

then, $\mathcal{A}$ satisfies that

$$
\lim _{\tau \rightarrow-\infty} e^{\mu \tau} \sup _{(v, w) \in \mathcal{A}(\tau)}\|(v, w)\|_{D\left(A_{1}\right)}^{2}=0 .
$$

Proof. From inequality (21), taking $t=\tau+3$ and $\varepsilon=2$, we have

$$
\begin{aligned}
& \left|\left(u_{m}^{\prime}(r), \gamma_{0}\left(u_{m}^{\prime}(r)\right)\right)\right|_{H}^{2} \\
\leq & (4 l+3) \int_{\tau+1}^{\tau+3}\left|\left(u_{m}^{\prime}(\theta), \gamma_{0}\left(u_{m}^{\prime}(\theta)\right)\right)\right|_{H}^{2} d \theta+\int_{\tau+1}^{\tau+3}\left(\left|h^{\prime}(\theta)\right|_{\Omega}^{2}+\left|\rho^{\prime}(\theta)\right|_{\partial \Omega}^{2}\right) d \theta
\end{aligned}
$$

for all $r \in[\tau+2, \tau+3]$, and any $m \geq 1$.

Analogously, if we take $s=\tau+1$ and $r=t=\tau+3$ in inequality (19), we have

$$
\begin{aligned}
& \int_{\tau+1}^{\tau+3}\left|\left(u_{m}^{\prime}(\theta), \gamma_{0}\left(u_{m}^{\prime}(\theta)\right)\right)\right|_{H}^{2} d \theta+\frac{\min \{1, \kappa\}}{1+\left\|\gamma_{0}\right\|^{2}}\left\|\left(u_{m}(\tau+3), \gamma_{0}\left(u_{m}(\tau+3)\right)\right)\right\|_{V_{1}}^{2} \\
& +2 \widetilde{\alpha}_{1}\left|u_{m}(\tau+3)\right|_{p, \Omega}^{p}+2 \widetilde{\alpha}_{1}\left|\gamma_{0}\left(u_{m}(\tau+3)\right)\right|_{p, \partial \Omega}^{p} \\
\leq & \max \{1, \kappa\}\left\|\left(u_{m}(\tau+1), \gamma_{0}\left(u_{m}(\tau+1)\right)\right)\right\|_{V_{1}}^{2}+\int_{\tau}^{\tau+3}\left(|h(\theta)|_{\Omega}^{2}+|\rho(\theta)|_{\partial \Omega}^{2}\right) d \theta \\
& +2 \widetilde{\alpha}_{2}\left|u_{m}(\tau+1)\right|_{p, \Omega}^{p}+2 \widetilde{\alpha}_{2}\left|\gamma_{0}\left(u_{m}(\tau+1)\right)\right|_{p, \partial \Omega}^{p}+4 \widetilde{\beta}(|\Omega|+|\partial \Omega|)
\end{aligned}
$$


for all $m \geq 1$.

From these two inequalities, we obtain

$$
\begin{aligned}
\left|\left(u_{m}^{\prime}(r), \gamma_{0}\left(u_{m}^{\prime}(r)\right)\right)\right|_{H}^{2} \leq & (4 l+3) \max \{1, \kappa\}\left\|\left(u_{m}(\tau+1), \gamma_{0}\left(u_{m}(\tau+1)\right)\right)\right\|_{V_{1}}^{2} \\
& +(4 l+3)\left(\int_{\tau}^{\tau+3}\left(|h(\theta)|_{\Omega}^{2}+|\rho(\theta)|_{\partial \Omega}^{2}\right) d \theta+4 \widetilde{\beta}(|\Omega|+|\partial \Omega|)\right) \\
& +2 \widetilde{\alpha}_{2}(4 l+3)\left(\left|u_{m}(\tau+1)\right|_{p, \Omega}^{p}+\left|\gamma_{0}\left(u_{m}(\tau+1)\right)\right|_{p, \partial \Omega}^{p}\right) \\
& +\int_{\tau+1}^{\tau+3}\left(\left|h^{\prime}(\theta)\right|_{\Omega}^{2}+\left|\rho^{\prime}(\theta)\right|_{\partial \Omega}^{2}\right) d \theta
\end{aligned}
$$

for all $r \in[\tau+2, \tau+3]$, and any $m \geq 1$.

Using this inequality and (25), it yields

$$
\begin{aligned}
& \left|\left(u_{m}^{\prime}(r), \gamma_{0}\left(u_{m}^{\prime}(r)\right)\right)\right|_{H}^{2} \\
\leq & C_{4}\left(\left|\left(u_{\tau}, \psi_{\tau}\right)\right|_{H}^{2}+\int_{\tau}^{\tau+3}\left(|h(\theta)|_{\Omega}^{2}+\left|h^{\prime}(\theta)\right|_{\Omega}^{2}+|\rho(\theta)|_{\partial \Omega}^{2}+\left|\rho^{\prime}(\theta)\right|_{\partial \Omega}^{2}\right) d \theta+1\right)
\end{aligned}
$$

for all $r \in[\tau+2, \tau+3]$, and any $m \geq 1$, where

$$
C_{4}=(4 l+3)\left(C_{3} \max \left\{1, \kappa, 2 \widetilde{\alpha}_{2}\right\}+\max \{1,4 \widetilde{\beta}(|\Omega|+|\partial \Omega|)\}\right) .
$$

From inequality (17), we obtain

$$
\begin{gathered}
\left|A_{1}\left(\left(u_{m}(r), \gamma_{0}\left(u_{m}(r)\right)\right)\right)\right|_{H}^{2} \leq 4\left|\left(u_{m}^{\prime}(r), \gamma_{0}\left(u_{m}^{\prime}(r)\right)\right)\right|_{H}^{2}+4 \max \left\{l, 4 c_{f g}^{2} c_{\partial \Omega}^{2}\right\}\left\|u_{m}(r), \gamma_{0}\left(u_{m}(r)\right)\right\|_{V_{1}}^{2} \\
+4 \kappa \beta|\Omega|+16 c_{f g}^{2}|\partial \Omega|+8\left(|h(r)|_{\Omega}^{2}+|\rho(r)|_{\partial \Omega}^{2}\right),
\end{gathered}
$$

where we have denoted $c_{\partial \Omega}$ the injection constant of $H^{1 / 2}(\partial \Omega)$ in $L^{2}(\partial \Omega)$.

Using this inequality, and thanks to (25) and (29), we obtain

$$
\begin{aligned}
& \left|A_{1}\left(\left(u_{m}(r), \gamma_{0}\left(u_{m}(r)\right)\right)\right)\right|_{H}^{2} \\
\leq & 4 C_{4}\left(\left|\left(u_{\tau}, \psi_{\tau}\right)\right|_{H}^{2}+\int_{\tau}^{\tau+3}\left(|h(\theta)|_{\Omega}^{2}+\left|h^{\prime}(\theta)\right|_{\Omega}^{2}+|\rho(\theta)|_{\partial \Omega}^{2}+\left|\rho^{\prime}(\theta)\right|_{\partial \Omega}^{2}\right) d \theta+1\right) \\
& +4 \max \left\{l, 4 c_{f g}^{2} c_{\partial \Omega}^{2}\right\} C_{3}\left(\left|\left(u_{\tau}, \psi_{\tau}\right)\right|_{H}^{2}+\int_{\tau}^{\tau+3}\left(|h(\theta)|_{\Omega}^{2}+|\rho(\theta)|_{\partial \Omega}^{2}\right) d \theta+1\right) \\
& +4 \kappa \beta|\Omega|+16 c_{f g}^{2}|\partial \Omega|+8\left(|h(r)|_{\Omega}^{2}+|\rho(r)|_{\partial \Omega}^{2}\right)
\end{aligned}
$$

for all $r \in[\tau+2, \tau+3]$, and any $m \geq 1$.

On the other hand, by Theorem 5 we have that $\left(u\left(\cdot ; \tau, u_{\tau}, \psi_{\tau}\right), \gamma_{0}\left(u\left(\cdot ; \tau, u_{\tau}, \psi_{\tau}\right)\right)\right) \in C([\tau+$ $\left.2, \tau+3] ; V_{1}\right)$. Since we know that $\left\{\left(u_{m}\left(\cdot ; \tau, u_{\tau}, \psi_{\tau}\right), \gamma_{0}\left(u_{m}\left(\cdot ; \tau, u_{\tau}, \psi_{\tau}\right)\right)\right)\right\}$ converges weakly to $\left(u\left(\cdot ; \tau, u_{\tau}, \psi_{\tau}\right), \gamma_{0}\left(u\left(\cdot ; \tau, u_{\tau}, \psi_{\tau}\right)\right)\right)$ in $L^{2}\left(\tau+2, \tau+3 ; D\left(A_{1}\right)\right)$, then, using Lemma 6 , and the above inequality, we have that

$$
\begin{gathered}
\left|A_{1}\left(\left(u(r), \gamma_{0}(u(r))\right)\right)\right|_{H}^{2} \leq C_{5}\left(\left|\left(u_{\tau}, \psi_{\tau}\right)\right|_{H}^{2}+\int_{\tau}^{\tau+3}\left(|h(\theta)|_{\Omega}^{2}+\left|h^{\prime}(\theta)\right|_{\Omega}^{2}+|\rho(\theta)|_{\partial \Omega}^{2}+\left|\rho^{\prime}(\theta)\right|_{\partial \Omega}^{2}\right) d \theta\right. \\
\left.+\sup _{r \in[\tau+2, \tau+3]}\left(|h(r)|_{\Omega}^{2}+|\rho(r)|_{\partial \Omega}^{2}\right)+1\right)
\end{gathered}
$$


for all $r \in[\tau+2, \tau+3]$, any $\tau \in \mathbb{R}$, and $\left(u_{\tau}, \psi_{\tau}\right) \in H$, where

$$
C_{5}=4 \max \left\{2, C_{4}+C_{3} \max \left\{l, 4 c_{f g}^{2} c_{\partial \Omega}^{2}\right\}+\kappa \beta|\Omega|+4 c_{f g}^{2}|\partial \Omega|\right\} .
$$

Now, observe that by the Cauchy-Schwartz inequality,

$$
|h(r)|_{\Omega} \leq|h(\tau+2)|_{\Omega}+\left(\int_{\tau+2}^{\tau+3}\left|h^{\prime}(\theta)\right|_{\Omega}^{2} d \theta\right)^{1 / 2}
$$

and

$$
|\rho(r)|_{\partial \Omega} \leq|\rho(\tau+2)|_{\partial \Omega}+\left(\int_{\tau+2}^{\tau+3}\left|\rho^{\prime}(\theta)\right|_{\partial \Omega}^{2} d \theta\right)^{1 / 2}
$$

for all $r \in[\tau+2, \tau+3]$.

Thus, from (30) and the above inequalities, we deduce that

$$
\begin{aligned}
\left\|U(\tau+2, \tau)\left(u_{\tau}, \psi_{\tau}\right)\right\|_{D\left(A_{1}\right)}^{2} \leq 3 C_{5} & \left(\left|\left(u_{\tau}, \psi_{\tau}\right)\right|_{H}^{2}+|h(\tau+2)|_{\Omega}^{2}+|\rho(\tau+2)|_{\partial \Omega}^{2}\right. \\
& \left.+\int_{\tau}^{\tau+3}\left(|h(\theta)|_{\Omega}^{2}+\left|h^{\prime}(\theta)\right|_{\Omega}^{2}+|\rho(\theta)|_{\partial \Omega}^{2}+\left|\rho^{\prime}(\theta)\right|_{\partial \Omega}^{2}\right) d \theta+1\right)
\end{aligned}
$$

for all $\tau \in \mathbb{R}$, and $\left(u_{\tau}, \psi_{\tau}\right) \in H$.

From this inequality and the fact that $\mathcal{A}(\tau)=U(\tau, \tau-2) \mathcal{A}(\tau-2)$, we obtain

$$
\begin{aligned}
\|(v, w)\|_{D\left(A_{1}\right)}^{2} \leq 3 C_{5} & \left(\sup _{(\varphi, \psi) \in \mathcal{A}(\tau-2)}|(\varphi, \psi)|_{H}^{2}+|h(\tau)|_{\Omega}^{2}+|\rho(\tau)|_{\partial \Omega}^{2}\right. \\
& \left.+\int_{\tau-2}^{\tau+1}\left(|h(\theta)|_{\Omega}^{2}+\left|h^{\prime}(\theta)\right|_{\Omega}^{2}+|\rho(\theta)|_{\partial \Omega}^{2}+\left|\rho^{\prime}(\theta)\right|_{\partial \Omega}^{2}\right) d \theta+1\right)
\end{aligned}
$$

for all $(v, w) \in \mathcal{A}(\tau)$, and any $\tau \in \mathbb{R}$.

Now, from this last inequality, and thanks to (7), (8), (26) and (27), we obtain (28).

Remark 15 Theorems 13 and 14 apply to the tempered pullback attractor $\mathcal{A}_{\mathcal{D}_{\mu}^{H}}$ and therefore also (trivially) to $\mathcal{A}_{\mathcal{D}_{F}^{H}}$ since $\mathcal{A}_{\mathcal{D}_{F}^{H}}(t) \subset \mathcal{A}_{\mathcal{D}_{\mu}^{H}}(t)$ for all $t \in \mathbb{R}$.

\section{Acknowledgments}

This work is dedicated to the memory of Prof. José Real, with our deepest and most sincere admiration, gratitude, and love. He was M.A. and P.M.-R.'s PhD-advisor, a clever mathematician with a sharp view on problems, a generous and wonderful colleague, and an even better friend. He passed away too soon, being only 60 years old. We miss him deeply, but he will stay forever in our hearts.

The authors thank the referee for observations that led us to improve the presentation of the manuscript.

This work has been partly supported by FEDER and Ministerio de Economía y Competitividad (Spain) under grant MTM2011-22411, and the Consejería de Innovación, Ciencia y Empresa (Junta de Andalucía), Proyecto de Excelencia P07-FQM-02468. 


\section{References}

[1] M. Altman, D. P. Altman, P. Ross, and H. Chang, The prediction of transient heat transfer performance of thermal energy storage devices, Chem. Eng. Progress Symposium Ser. 61 (1965), 289-297.

[2] M. Anguiano, T. Caraballo, and J. Real, $H^{2}$-Boundedness of the pullback attractor for a non-autonomous reaction-diffusion equation, Nonlinear Anal. 72 (2010), 876-880.

[3] M. Anguiano, T. Caraballo, and J. Real, An exponential growth condition in $H^{2}$ for the pullback attractor of a non-autonomous reaction-diffusion equation, Nonlinear Anal. 72 (2010), 4071-4076.

[4] M. Anguiano, P. Marín-Rubio, and J. Real, Pullback attractors for non-autonomous reaction-diffusion equations with dynamical boundary conditions, J. Math. Anal. Appl. 383 (2011), 608-618.

[5] H. Amann and J. Escher, Strongly continuous dual semigroups, Ann. Mat. Pura Appl. (4) 171 (1996), 41-62.

[6] J. M. Arrieta, P. Quittner, and A. Rodríguez-Bernal, Parabolic problems with nonlinear dynamical boundary conditions and singular initial data, Differential Integral Equations 14 (2001), 1487-1510.

[7] W. F. Bauer, Modified Sturm-Liouville systems, Quart. Appl. Math. 11 (1953), 273-283.

[8] I. Bejenaru, J. I. Díaz, and I. I. Vrabie, An abstract approximate controllability result and applications to elliptic and parabolic systems with dynamic boundary conditions, Electron. J. Differential Equations 2001 (2001), 19 pp.

[9] T. Caraballo, G. Łukaszewicz, and J. Real, Pullback attractors for asymptotically compact non-autonomous dynamical systems, Nonlinear Anal. 64 (2006), 484-498.

[10] T. Caraballo, G. Łukaszewicz, and J. Real, Pullback attractors for non-autonomous 2D Navier-Stokes equations in unbounded domains, C. R. Math. Acad. Sci. Paris 342 (2006), 263-268.

[11] C. Cavaterra, C. G. Gal, M. Grasselli, and A. Miranville, Phase-field systems with nonlinear coupling and dynamic boundary conditions, Nonlinear Anal. 72 (2010), 2375-2399.

[12] I. Chueshov and B. Schmalfuß, Qualitative behavior of a class of stochastic parabolic PDES with dynamical boundary conditions, Discrete Contin. Dyn. Syst. 18 (2007), 315338.

[13] A. Constantin and J. Escher, Global existence for fully parabolic boundary value problems, NoDEA Nonlinear Differential Equations Appl. 13 (2006), 91-118.

[14] J. Crank, The Mathematics of Diffusion, Clarendon Press, Oxford, 1975.

[15] H. Crauel, A. Debussche, and F. Flandoli, Random attractors, J. Dynam. Differential Equations 9 (1997), 307-341.

[16] J. Escher, Quasilinear parabolic systems with dynamical boundary conditions, Comm. Partial Differential Equations 18 (1993), 1309-1364. 
[17] J. Escher, On the qualitative behaviour of some semilinear parabolic problems, Differential Integral Equations 8 (1995), 247-267.

[18] Z. H. Fan and C. K. Zhong, Attractors for parabolic equations with dynamic boundary conditions, Nonlinear Anal. 68 (2008), 1723-1732.

[19] W. Feller, The parabolic differential equations and the associated semi-groups of transforms, Ann. of Math. 55 (1952), 468-519.

[20] J. Filo and S. Luckhaus, Modelling surface runoff and infiltration of rain by an ellipticparabolic equation coupled with a first-order equation on the boundary, Arch. Ration. Mech. Anal. 146 (1999), 157-182.

[21] A. Friedman and M. Shinbrot, The initial value problem for the linearized equations of water waves, J. Math. Mech. 17 (1967), 107-180.

[22] C. G. Gal and A. Miranville, Uniform global attractors for non-isothermal viscous and non-viscous Cahn-Hilliard equations with dynamic boundary conditions, Nonlinear Anal. Real World Appl. 10 (2009), 1738-1766.

[23] C. Gal and M. Warma, Well posedness and the global attractor of some quasi-linear parabolic equations with nonlinear dynamic boundary conditions, Differential Integral Equations 23 (2010), 327-358.

[24] J. García-Luengo, P. Marín-Rubio, and J. Real, $H^{2}$-boundedness of the pullback attractors for non-autonomous 2D Navier-Stokes equations in bounded domains, Nonlinear Anal. 74 (2011), 4882-4887.

[25] G. R. Goldstein, Derivation and physical interpretation of general boundary conditions, Adv. Differential Equations 11 (2006), 457-480.

[26] A. E. Green and K. A. Lindsay, Thermoelasticity, J. Elasticity, 2 (1972), 1-7.

[27] T. Hintermann, Evolution equations with dynamic boundary conditions, Proc. Roy. Soc. Edinburgh Sect. A 113 (1989), 43-60.

[28] R. E. Langer, A problem in diffusion or in the flow of heat for a solid in contact with a fluid, Tohoku Math. J. 35 (1932), 260-275.

[29] L. Lapidus and N. R. Amundson, Editors, Chemical Reactor Theory, Prentice-Hall, Englewood Cliffs, 1977.

[30] I. Lasiecka and N. Fourrier, Regularity and stability of a wave equation with a strong damping and dynamic boundary conditions, Evol. Equ. Control Theory 2 (2013), 631667.

[31] J. L. Lions, Équations différentielles opérationelles et problèmes aux limites, SpringerVerlag, Berlin, 1961.

[32] J. L. Lions, Quelques Méthodes de Résolution des Problèmes aux Limites Non linèaires, Dunod, Paris, 1969. 
[33] G. Pincet-Mailly and J.-F. Rault, Nonlinear convection in reaction-diffusion equations under dynamical boundary conditions, Electron. J. Differential Equations 2013 (2013), $14 \mathrm{pp}$.

[34] H. W. March and W. Weaver, The diffusion problem for a solid in contact with a stirred liquid, Physical Review 31 (1928), 1072-1082.

[35] P. Marín-Rubio and J. Real, On the relation between two different concepts of pullback attractors for non-autonomous dynamical systems, Nonlinear Anal. 71 (2009), 3956-3963.

[36] W. Peddie, Note on the cooling of a sphere in a mass of well stirred liquid, Proc. Edinburgh Math. Soc. 19 (1901), 34-35.

[37] R. L. Peek, Solutions to a problem in diffusion employing a non-orthogonal sine series, Ann. of Math. 30 (1928/29), 265-269.

[38] L. Popescu and A. Rodríguez-Bernal, On a singularly perturbed wave equation with dynamic boundary conditions, Proc. Roy. Soc. Edinburgh Sect. A 134 (2004), 389-413.

[39] M. Primicerio and J.F. Rodrigues, The Hele-Shaw problem with nonlocal injection condition, in Nonlinear Mathematical Problems in Industry, pp. 375-390, GAKUTO, Tokyo, 1993.

[40] J. Prüss, R. Racke, and S. Zheng, Maximal regularity and asymptotic behavior of solutions for the Cahn-Hilliard equation with dynamic boundary conditions, Ann. Mat. Pura Appl. (4) 185 (2006), 627-648.

[41] J. Prüss and M. Wilke, Maximal $L^{p}$-regularity and long-time behaviour of the nonisothermal Cahn-Hilliard equation with dynamic boundary conditions, in Partial differential equations and functional analysis, pp. 209-236, Oper. Theory Adv. Appl. 168, Birkhäuser, Basel, 2006.

[42] S. Selberher, Analysis and Simulation of Semiconductor Devices, Springer, Wien, 1984.

[43] V. A. Solonnikov and E. V. Frolova, $L_{p}$-theory for the Stefan problem, J. Math. Sci. 99 (2000), 989-1006.

[44] R. Temam, Infinite-Dimensional Dynamical Systems in Mechanics and Physics, Springer, New York, 1988.

[45] J. L. Vázquez and E. Vitillaro, Heat equation with dynamical boundary conditions of reactive-diffusive type, J. Differential Equations 250 (2011), 2143-2161.

[46] L. Yang, Uniform attractors for the closed process and applications to the reactiondiffusion equation with dynamical boundary condition, Nonlinear Anal. 71 (2009), 40124025 .

[47] L. Yang and M. Yang, Long-time behavior of reaction-diffusion equations with dynamical boundary condition, Nonlinear Anal. 74 (2011), 3876-3883.

[48] L. Yang, M. Yang, and P.E. Kloeden, Pullback attractors for non-autonomous quasilinear parabolic equations with dynamical boundary conditions, Discrete and Continuous Dynamical Systems Series B, 17 (2012), 2635-2651. 